\title{
Coupled multi-physics simulation of chloride diffusion in saturated and unsaturated concrete
}

\author{
Ying Zhang ${ }^{\mathrm{a}}$, Giovanni Di Luzio ${ }^{\mathrm{b}}$, Mohammed Alnaggar ${ }^{\mathrm{a}, *}$ \\ ${ }^{a}$ Rensselaer polytechnic Institute, Troy, NY, USA \\ ${ }^{b}$ Politecnico Di Milano, Milan, Italy
}

\begin{abstract}
Chloride-induced corrosion of steel reinforcement is one of the major long-term deterioration mechanisms for reinforced concrete infrastructures. Chloride transport through cement-based materials is a complex chemo-physical process involving ionic diffusion in concentrated solution, pore structure, chemistry, membrane permeability of the matrix, cracking, and the variation of the internal and external environmental conditions. Although in the literature there are plenty of both simplistic phenomenological models and sophisticated models, in this study, a new model is developed taking aim at capturing the fundamental physics and, at the same time, having a formulation as simple as possible that it can be effectively calibrated and validated using available limited experimental data. The model couples the ionic diffusion process with the concrete micro-structure evolution due to continued hydration accounting for hygro-thermal variations and their effects on both the diffusion and hydration processes. The formulation is implemented in a semi-discrete conduit transport network that mimics the internal heterogeneity of the cementitious material by connecting the matrix space between coarse aggregate pieces. This allows the model to replicate naturally the meso-scale tortuosity effect which is an important feature towards representing realistically the heterogeneity-induced variations of chloride concentration within the concrete. The limited model parameters are carefully calibrated and the formulation is validated by simulating multiple experiments ranging from diffusion through pastes to large concrete cylinders. The results of numerical simulations show the ability of the model to describe spatial and temporal evolution of the chloride concentration within the samples under varying chloride concentrations and temperature boundary conditions within both saturated and
\end{abstract}


unsaturated concrete.

Keywords: Chloride transport, Concrete pore structure, Aging and deterioration, Multi-physics modeling

\section{INTRODUCTION}

Chloride penetration in concrete is one of the major causes of long-term corrosion of steel re3 inforcement in reinforced concrete structures and, particularly in infrastructure exposed to chlo-

4 ride rich environments, such as marine structures and roadways subjected to deicing salts [1]. In 5 many studies, to predict service life of reinforced concrete structures, such as bridges, the pene6 tration depth of chloride is utilized as measurement of long-term corrosion time-to-failure [2, 3].

7 The evaluation of chloride diffusion in concrete is also important in order to develop new durable 8 concrete and to assess the durability of structures especially under extremely aggressive environ9 ments; for instance, this is the key activity of the project ReSHEALience (www.uhdc.eu) [4].

In past years, chloride transport in concrete has drawn much attention and many methods 11 for determining chloride diffusivity in both saturated and non-saturated conditions have been 12 developed $[5,6]$. The early attempts to simulate chloride penetration into concrete were focused 13 on describing the ion diffusion in concentrated solution, such as Chatterji that firstly proposed the 14 fundamental equations of diffusion by coupling Fick's laws, Nernst and Nernst-Planck equations 15 together [7, 8, 9]. Bažant [10] applied Maxwell's equations of electrostatics to consider the 16 balance of electric charge. Then Zhang and Gjørv [11] introduced the effects of ionic species 17 activity by expressing the driving diffusion force based on chemical potentials but not on the 18 species concentration. Samson and Marchand [12] showed that the total membrane potential is 19 two times larger when considering the chemical activity by analyzing the numerical solution of 20 Extended Nernst-Planck Model and, therefore, its effect needs to be also considered as a diffusion 21 driving force.

\footnotetext{
${ }^{*}$ Corresponding author

Email addresses: zhangy61erpi .edu (Ying Zhang), giovanni.diluzio@polimi.it (Giovanni Di Luzio), a lnagm2 erpi . edu (Mohammed Alnaggar) 
These early studies performed thorough analysis on the influence of the concentrated solution chemistry on the diffusion of ionic species. Based on that, later studies moved forward to explore the effect of the complex concrete micro-structure and its evolution since chloride transport in concrete pore solution differs from that in concentrated solution and pore structure and chemical reactions have significant influence on the diffusion process. Tang [13, 14] studied the concentration dependence of diffusion and migration of chloride ions in pore solution by considering the effect of surrounding ions and membrane potential. Masi et al. [15] simulated the diffusion process by means of the percolation concepts. System porosity and the adsorption parameter were included in the model. Starting from Fick's law, Xi and Bažant [16] deduced a general expression for chloride diffusion including a series of factors, such as adsorption isotherm, temperature influence, aggregate effects and concentration dependence. Johannesson et al. [17] modelled the multi-species ionic diffusion in concrete with account to interaction between ions in the pore solution and the cement hydrates. Nguyen and Amiri investigated the electrical double layer (EDL) effects on chloride transport and found that the EDL is strong in concrete containing slag, while relatively weak in regular concrete $[18,19]$. Li et al. [20] proposed a multi-phase model for predicting the effective coefficient of chlorides in concrete and discussed the effect of aggregates, showing that the size and shape of aggregate have small influence on the diffusion of chloride. Marchand et al. proposed a phenomenological model considering the change of porosity over time [21]. This model was later improved by considering the effect of temperature on ionic transport in cementitious materials through energy balance [22, 20].

In recent years, a few advanced models have been proposed, aiming at capturing the features of chloride transport in complex and variable exposure conditions. Based on the experimental results, Isteita and $\mathrm{Xi}$ studied the coupling effect between chloride penetration and temperature gradient and proposed a coupling parameter to consider the effect of temperature variation on chloride penetration [23]. Similarly, a fitting parameter coupling moisture transport and chloride penetration is presented in [24]. Ožbolt et al proposed a 3D chemo-hyrgo-thermo mechanical model for continuum that is able to simulate physical processes before [25, 26] and after [27] depassivation of steel reinforcement. Song et al proposed the concept of "contact duration" to 
consider the time dependence of chloride binding capacity [28]. Conciatori et al. performed sensitivity analysis to quantify the influence of parameter variation [29]. The results showed that chloride ingress is very sensitive to many parameters including porosity, temperature, ionic diffusion coefficient, water permeability etc., and exhibits high variability at the early period of the ingress. This indicates that modelling chloride penetration with too many parameters is very challenging in calibration and might results in unsuccessful fitting.

As it can be seen from this short review, phenomenological models are not accurate enough and are limited to the range of data to which they were fitted and thus are not effectively predictive. Simple theoretical models consider a subset of the main governing processes that affect chloride penetration and thus, can not be generalized over a wide range of exposure conditions. Other sophisticated models consider a large number of parameters which are hard to calibrate uniquely using limited experimental data. In this paper, a new model is developed with the aim to strike a sensitive balance between capturing the fundamental physics, but at the same time, having a formulation as simple as possible without compromising its versatility and generality to achieve effective calibration and successful validation. Moreover, the proposed formulation is based on a discrete approach that considers many significant features presented in the next section, such as accounting for the heterogeneity induced by coarse aggregate through the use of a discrete lattice system that represents the flow within the meso-scale structure.

\section{Significance of the proposed research}

This model is formulated by considering five major chemo-physical phenomena governing chloride ion diffusion in cementitious materials, namely, 1) effects of chemical activity and membrane potential on ion transport in concentrated solutions in porous media, 2) effects of pore structure evolution (pore volume and tortuosity) on diffusivity, 3) effects of surrounding ions that make the diffusivity concentration-dependent, 4) the thermal effects on diffusivity, chloride binding capacity, and micro-structure change as reflected by cementitious material hydration reactions, and 5) effects of advection due to moisture transport in unsaturated conditions. During the development of this model, the authors decided not to explicitly model the phenomena that 
are hard to measure experimentally, controversial or have very limited experimental data that represent it. Instead, for such phenomena, simplifying assumptions broadly supported in literature were adopted. This approach is the key in striking the balance between simplicity and representation of chemo-physical phenomena in this model. For example, the model does not represent explicitly the diffusion of all ionic species present in the pore solution. Instead, it represents their effects on chloride diffusion following simplified counter ion effects formulations with very limited parameters. Theoretically, one could model the diffusion of all existing species in the solution and impose electric charge balance. Yet practically, this means that experimental data relevant to rapid chloride penetration tests will not be sufficient to calibrate the various diffusing species and the concentrations of these species have to be explicitly monitored to correctly identify their diffusion parameters. If such comprehensive experimental data is not available, model parameters relevant to all other diffusing species will be assumed and correspondingly, their effects on the chloride diffusion modeling will be high. In this case, while there should be some additional benefits from modeling the various diffusion processes, the biased parameter identification would reduce the model robustness and increase its sensitivity. Additionally, the model neglects the time dependence of the chloride binding process because evaluating its rate requires modeling of phenomena at the level of material gel microstructure which is much finer than the scale at which the model is formulated, and it is also much finer than the scale at which the chloride concentrations are determined in the experiments used for calibration and validation. Therefore, chloride binding to the matrix is considered including the effects of temperature on the binding capacity, but the local variation of the binding capacity over time is ignored.

As a result, model free parameters are limited yet, the modeled chemo-physical processes are comprehensive enough to represent a wide range of experimental results. In addition, the model is readily extendable to multi-ionic diffusion with full charge balance if comprehensive experimental data are made available as it is implemented within a fully coupled multi-physics framework that accounts for moisture and heat diffusion along with the typical cementitious material chemical reactions.

Geometrically speaking, one of the main advantages of the proposed model is that it is imple- 
mented in a discrete lattice system that represents flow through the concrete internal structure. Again, when experimental data is made available in which, the chloride concentration in the cementitious matrix is separated from that of the aggregate within it, this model can better represent the variations of concentration due to the heterogeneity of the concrete internal structure considering the cement matrix as shortcut for flow as compared to aggregate locations. Nevertheless, given the fact that this model advances the flow in between the matrix zones inside the concrete structure, it naturally captures the meso-scale tortuosity. This is a very important aspect when dealing with the formation of micro-cell corrosion, i.e. position of anodic and cathodic sites. Chloride ions ingress in a tortuous way that avoids aggregate as it is hard to penetrate, leading to a non-uniform distribution of chloride concentration at any depth. Given the fact that the formation of micro-cell corrosion depends on local concentration rather than average concentration, as predicted by most existing models, it is of great importance to characterize the meso-scale tortuosity when modeling the chloride-induced corrosion in concrete. This feature is beyond the reach of any macroscopic continuum model since it inherently homogenizes the concrete internal structure. Furthermore, this discrete nature makes the model readily extendable to meso-structural cracking effects necessary to model the two-way coupling between cracking on diffusivity and cracking evolution under corrosion progression. This two-way coupling has been already employed in modeling concrete spalling at elevated temperatures [30] and moisture diffusion behavior of self-healing concrete [31].

Certainly, the benefits of the meso-scale discrete discretization approach must be paid with an additional computational cost. However, the proposed approach does not add a very significant computational cost when compared to its continuum counterparts. The reason for this is twofold: (1) continuum meshes for diffusion problems must have very fine discretization meshes at the boundaries to capture the high gradients with an acceptable accuracy; (2) continuum meshes would discretize all the space around and within the aggregates with nearly the same density. For these reasons, the discrete approaches are more effective compared to continuum approaches, yet of course they are more computationally demanding. Based on the authors experience in using both the discrete (1D conduit) scheme and the FE continuum (3D tetrahedral elements) scheme 
in many previous research applications concerning aging, creep, shrinkage and Alkali Silica Reaction (ASR) problems $[32,33,34,35,36]$, they noticed that the discrete mesh requires no more than $50 \%$ of computational time compared to continuum mesh with similar refinement.

This difference has not been quantitatively evaluated but the authors have consistently observed it in their work.

Finally, in this model, aging as well as heat and moisture transport processes are introduced by using a comprehensive hygro-thermo-chemical model with which the new chloride diffusion model is fully coupled. As a result, the effects of the changes in water to cement ratio $(w / c)$, change in porosity over time, presence of thermal gradients or temperature variations, and diffusion in unsaturated concrete are naturally captured without introducing additional fitting parameters. While it is true that aging and hygro-thermal variations may not be of significant effect on chloride diffusion in some field applications where structures are old enough and hygro-thermal conditions are nearly constant, the importance of considering these features stems from the need to use accelerated experimental data in which all or part of these aging and hygro-thermal effects are very significant. Without their proper considerations, model predictions of real-world situations that are based on accelerated lab testing can be considerably inaccurate.

\section{Multi-physics formulation}

Many factors influence the ionic transport in cementitious materials. However, since it is mainly taking place within the liquid water in the pore structure, the physics of ionic transport can start from considering the diffusion of ions in concentrated solutions (liquid pore solution) and then account for the effects of the pore structure. These main effects can be summarized as

(1) Geometrical effect, i.e. pore size distribution and its connectivity.

(2) Chemical reactions effect, since the pore walls are chemically active, part of the moving ions tend to bind with the pore walls.

(3) Electric potential effect, since the pore walls are not neutral but in general negatively charged, a membrane potential is generated and affects the diffusion of ionic species. 
It is also important to consider that the internal pore structure of a cementitious material ages and changes in time. As a result of the chemical reactions, including hydration, polymerization and gel densification [37], the pore structure densifies, and, in turn, results in time-dependent decreasing porosity and increasing tortuosity. In addition, if the ionic solution is not in thermodynamic equilibrium (i.e. there is moisture gradient within the pores), then advection of the dissolved ions has to be also considered. Moreover, all the processes mentioned above are affected by thermal conditions, i.e. as temperature increases, ionic mobility, moisture diffusivity, chemical reactions rates and binding capacities usually increase too.

To predict accurately the transport of an ionic species in a cementitious material one has to consider all the above mentioned phenomena. However, as discussed earlier in Sec. 2, the approach used here in developing the proposed formulation accounts for the most relevant electrochemo-physical phenomena encountered during chloride penetration using the minimum amount of free parameters that can be calibrated from standard tests at the macro-scale.

In the following sections, the formulation of a chloride diffusion model will be detailed. Since the model depends on temperature and moisture variations, as well as, on material aging, these phenomena are here modeled using the Hygro-thermo-chemical (HTC) model by Di Luzio and Cusatis $[38,39]$ which will be briefly reviewed and the coupling between the chloride diffusion model and the HTC model will be also presented.

\subsection{Formulation of chloride diffusion}

The macroscopic equation for the transport of ionic species can be based on the extended Nernst-Planck equation with an advection term [40, 41, 42, 43]. The mass balance equation (continuity equation) of chloride ions can be written as

$$
\frac{\partial}{\partial t}\left(\frac{w_{c a p}}{\rho_{w}} C+w_{s} C_{b}\right)=-\boldsymbol{\nabla} \cdot J_{c}+\frac{w_{c a p}}{\rho_{w}} \dot{r}
$$

where $C$ is the concentration of the ionic species in the pore solution $\left(\mathrm{mol} / \mathrm{m}^{3}\right), w_{\text {cap }}$ is the mass of capillary water content $\left(\mathrm{kg} / \mathrm{m}^{3}\right), \rho_{w}$ is the water density $\left(\mathrm{kg} / \mathrm{m}^{3}\right), w_{c a p} / \rho_{w}$ is the volumetric water content $\left(\mathrm{m}^{3} / \mathrm{m}^{3}\right), w_{s}$ is the volumetric solid content $\left(\mathrm{m}^{3} / \mathrm{m}^{3}\right), C_{b}$ is the concentration of 
the species bound in the solid content $\left(\mathrm{mol} / \mathrm{m}^{3}\right), J_{c}$ is the ionic flux $\left(\mathrm{mol} / \mathrm{m}^{2} / \mathrm{s}\right)$ in cementitious materials, $\dot{r}$ stands for chemical reaction rate and $\frac{w_{c a p}}{\rho_{w}} \dot{r}$ is a source/sink term accounting for the creation/consumption of the ions in solution as a result of chemical reactions $\left(\mathrm{mol} / \mathrm{m}^{3} / \mathrm{s}\right)$. It should be noted here that chloride ions are assumed to move only within the capillary pores occupied by capillary water $w_{\text {cap }}$ in this formulation. As it will be explained later, the HTC model differentiates between capillary and physically bound (adsorbed) water which together form the non-evaporable water. This assumption is very reasonable since the physically bound water is adsorbed in the the C-S-H gel structure and is nearly immobile. As will be shown in the simulation results, this assumption will be validated by modeling diffusion in unsaturated conditions with calibrated model parameters using saturated conditions only.

There are two different types of chemical reactions: the reactions occurring solely in the aqueous phase (i.e. formation or dissociation of acids) and the reactions that involve more than one phase, such as surface or classical reactions. The adsorption of ions, i.e. the capture of ions by the surface of the solid as a result of electrostatic forces, is a surface reaction, whereas dissolution or precipitation reactions belong to the classical category of chemical reactions. In Eq. 1, the second term in the LHS corresponds to the surface or classical chemical reactions, on the contrary the last term in the RHS considers reactions occurring solely in the aqueous phase. However, since the hydrated cement paste has a highly reactive nature, we can assume that the surface or classical reactions are much more significant than reactions occurring solely in the aqueous phase. Expanding Eq. 1 and neglecting the chemical reactions in the aqueous phase $(\dot{r}=0)$ one gets

$$
\frac{C}{\rho_{w}} \frac{\partial w_{c a p}}{\partial t}+\frac{w_{c a p}}{\rho_{w}} \frac{\partial C}{\partial t}+w_{s} \frac{\partial C_{b}}{\partial t}+C_{b} \frac{\partial w_{s}}{\partial t}=-\nabla \cdot J_{c}
$$

where only dissolution/precipitation reactions are taken into account.

The so-called chloride binding isotherm provides the relationship between the free and the bound chloride concentration at a given temperature. In the literature four types of adsorption isotherm (linear, Langmuir, Freundlich and BET binding isotherm) have been proposed [44] and among them the Freundlich isotherm appears to fit the experimental results very well $[16,44,45$, 
46]. Therefore, the Freundlich isotherm is adopted in this study and has the following expression

$$
\log \left(C_{b}^{\prime}\right)=A_{0} \log (C)+B_{0}
$$

$$
C_{b}=\frac{C^{A_{0}} 10^{B_{0}} \rho_{\mathrm{s}}}{1000 M_{\mathrm{Cl}}} \exp \left[\frac{U_{b}}{R}\left(\frac{1}{T_{0}}-\frac{1}{T}\right)\right]
$$
parameters. isotherm in Eq. 3 can be rewritten as

$$
C_{b}=\frac{C^{A_{0}} 10^{B_{0}} \rho_{\mathrm{S}}}{1000 M_{\mathrm{Cl}}}
$$
dependent coefficient

where $C_{b}^{\prime}$ is the mass of bound chlorides per gram of solid gel (mg/g), $A_{0}$ and $B_{0}$ are two material

Because the relationship between $C_{b}^{\prime}$ and $C_{b}$ can be expressed as $C_{b}^{\prime}=1000 M_{\mathrm{Cl}} C_{b} / \rho_{s}$, where $M_{\mathrm{Cl}}$ is the molar mass of chloride, $\rho_{s}$ is the density of solid content $\left(\mathrm{kg} / \mathrm{m}^{3}\right)$, the Freundlich

The binding process is also influenced by the temperature through different mechanisms. There are two main types of binding processes: (1) the physical binding that is caused by the adsorption of chloride ions on the C-S-H surface; (2) the chemical binding that leads to the formation of Friedel's salt [44]. Temperature variation affects these two binding processes differently. As temperature increases, the physical binding process slows down because of higher thermal energy of ions. On the contrary, higher temperature accelerates the chemical binding process [47], although the increased temperature increases the solubility of Friedel's salt [48]. For temperature variations between $0 \sim 50^{\circ}$ and for low chloride concentration $(\leq 1 \mathrm{~mol} / \mathrm{L})$, a significant increase of bound chloride is observed in experimental data as temperature rises $[49,50,51]$. Other experiments report that there is no noticeable increase of the Friedel's salt solubility [52] and the concentration of bound chloride shows slight differences as temperature varies $[48,51,53]$. Consequently, in this study, to describe the effects of temperature on chloride binding, only the effect of chemical binding is considered using an exponential temperature-

where $U_{b}$ is a coefficient that characterized the temperature effect on the adsorption process, $A_{0}$ 
and $B_{0}$ are both determined at the reference temperature $T_{0}$. The temporal derivative of the bound chloride from Eq. 5 can be expressed as

$$
\frac{\partial C_{b}}{\partial t}=\frac{\partial C_{b}}{\partial C} \frac{\partial C}{\partial t}+\frac{\partial C_{b}}{\partial T} \frac{\partial T}{\partial t}=\frac{A_{0} C_{b}}{C} \frac{\partial C}{\partial t}+\frac{U_{b} C_{b}}{R T^{2}} \frac{\partial T}{\partial t}
$$

The sorption isotherm law is needed to determine the capillary water content, $w_{\text {cap }}$, as a function of the relative humidity and aging. In the HTC model $w_{\text {cap }}$ is formulated using an improved version of the original expression proposed by Norling Mjornell [54]. Based on this sorption isotherm formulation, the capillary water in concrete can be expressed as a function of relative humidity, $h$, and hydration degree $\alpha_{c}$. The volumetric solid content, $w_{s}$, can be calculated as $\left(c \alpha_{c}+w_{n}\right) / \rho_{s}$, where $c$ is the mass of cement content $\left(\mathrm{kg} / \mathrm{m}^{3}\right), \alpha_{c}$ is the hydration degree, $w_{n}$ is the non-evaporable water content $\left(\mathrm{kg} / \mathrm{m}^{3}\right)$. The temporal derivative of $w_{c a p}$ and $w_{s}$ an be written as

$$
\frac{\partial w_{c a p}}{\partial t}=\frac{\partial w_{c a p}}{\partial h} \frac{\partial h}{\partial t}+\frac{\partial w_{c a p}}{\partial \alpha_{c}} \dot{\alpha}_{c} \quad \text { and } \quad \frac{\partial w_{s}}{\partial t}=\left(c \dot{\alpha}_{c}+\dot{w}_{n}\right) / \rho_{s}
$$

Substituting Eqs. 6-7 into Eq. 2, the LHS of Eq. 2 can be expressed as

$$
\begin{aligned}
\frac{C}{\rho_{w}} \frac{\partial w_{c a p}}{\partial t}+\frac{w_{c a p}}{\rho_{w}} \frac{\partial C}{\partial t}+w_{s} \frac{\partial C_{b}}{\partial t}+C_{b} \frac{\partial w_{s}}{\partial t}=\left(\frac{w_{c a p}}{\rho_{w}}+\frac{w_{s} A_{0} C_{b}}{C}\right) \frac{\partial C}{\partial t}+ \\
\left(c \dot{\alpha}_{c}+\dot{w}_{n}\right) \frac{C_{b}}{\rho_{s}}+\frac{C \dot{\alpha}_{c}}{\rho_{w}}\left(\frac{\partial w_{c a p}}{\partial \alpha_{c}}\right)+\frac{w_{s} U_{b} C_{b}}{R T^{2}} \frac{\partial T}{\partial t}+\frac{C}{\rho_{w}} \frac{\partial w_{c a p}}{\partial h} \frac{\partial h}{\partial t}
\end{aligned}
$$

For fully saturated conditions with $h=1$, the last term in Eq. 8 is equal to zero. The various quantities, $w_{c a p}, w_{n}, \alpha_{c}$, and $h$ can be obtained by coupling Eq. 2 with the HTC model [38, 39] (see Section 4).

In the RHS of Eq. 2, we need now to specify the term, $J_{c}$, which represents the flux of chloride ions in the cementitious material. In the transport of charged ions through a porous media like concrete, its pore structure, characterized by porosity, pores shape and spatial distribution, connectivity, tortuosity, as well as, the interfacial transition zone (ITZ), plays an important role [55]. 
For partially saturated conditions, moisture movement occurs, leading to the motion of water from higher saturated pores toward less saturated pores. This means that ionic species can also be transported along with the fluid by advection. Therefore, the flux of ionic species in concrete pore solution can be expressed as

$$
J_{c}=f_{\phi} J_{s}+v C \quad \text { with } \quad J_{s}=f_{\gamma} f_{\psi} f_{s} f_{T} J_{0}
$$

where $J_{s}$ is the flux of ionic species in concentrated solution of a porous material, $f_{\phi}$ is the function that considers the effect of pore structure and $v$ is the fluid mass velocity. For fully saturated conditions, moisture inside concrete is in static equilibrium and thus no moisture mass transport occurs (i.e. $v=0$ ). The flux of ionic species in concentrated solution is very different from the flux in dilute solution due to the interactions of surrounding ions and the existence of an electro-chemical potential. The flux in dilute solution can be expressed with the first Fick's law as $J_{0}=-D_{0} \nabla C$, in which $D_{0}$ is the diffusivity coefficient in dilute solution. The flux of concentrated solution, $J_{s}$, can be related to $J_{0}$ through a set of coefficients: $f_{\gamma}, f_{\psi}, f_{s}$ and $f_{T}$, which account for the different chemo-physical phenomena encountered in the diffusion process of a concentrated solution with respect to a dilute solution. These phenomena, and the corresponding coefficients that represent them, are explained hereafter.

\subsection{Effect of pore solution chemistry}

Since the pore solution of a cement paste is very rich in ions with an initially very high alkaline concentration [56], the transport of ionic species is driven by the gradient of electrochemical potential [57], which can be written as

$$
u=u_{0}+R T \ln (\gamma C)+z F \psi
$$

where $u$ is the electro-chemical potential of a species $(\mathrm{J} / \mathrm{mol}), u_{0}$ is the chemical potential in the standard state, $\gamma$ is the activity coefficient $(-), z$ is the valency of the ion, $F$ is the Faraday constant $(96485.33 \mathrm{C} / \mathrm{mol}), R$ is the ideal gas constant $(8.3143 \mathrm{~J} / \mathrm{mol} / \mathrm{K}), T$ is the temperature of the material $(\mathrm{K})$, and $\psi$ is the electrical potential $(\mathrm{V})$. In pore solution, the electrical potential 
$\psi$ consists of two components. The first represents the formed counter-electrical potential $\psi_{0}$ between the moving chloride ions and the surrounding moving cations. The second is called a membrane-electrical potential $\psi_{m}$ and is caused by membrane effects of cement paste. Both electrical potentials produce an opposite force and are combined together to form the overall electro-chemical potential.

The counter-electrical potential, $\psi_{0}$, is the result of the difference in drift velocities between cations and anions [13] and can be expressed as

$$
\psi_{0}=-\frac{R T}{F}\left(\tau_{+0}-\tau_{-0}\right) \ln (\gamma C)=-\frac{R T}{F} K_{\tau 0} \ln (\gamma C)
$$

where $\tau_{+0}$ and $\tau_{-0}$ are the transference number of cations and anions, and the symbol $K_{\tau 0}$ denotes the difference in transference number or drift velocity in the pure solution. For a specific electrolytic solution, it can be regarded as constant, e.g. for sodium chloride solution, $K_{\tau 0}=-0.207[14]$.

The membrane-electrical potential, $\psi_{m}$, is induced by moving within the C-S-H gel porous structure which has a negatively charged surface that behaves as an electro-negative membrane: accelerating cations and decelerating anions. As a result, there is a selective permeability which increases the difference in mobility between cations and anions. The $\psi_{m}$ can be expressed as $[13,58]$

$$
\psi_{\mathrm{m}}=-\frac{R T}{F} K_{\tau \mathrm{m}} \ln (\gamma C)
$$

where $K_{\tau \mathrm{m}}$ denotes the difference in mobility caused by the membrane-electrical potential. The resulting electrical potential in pore solution, $\psi=\psi_{0}+\psi_{m}$, can be substituted in Eq. 10 which gives the total electro-chemical potential as:

$$
u=u_{0}+R T f_{\psi} \ln (\gamma C) \quad \text { with } \quad f_{\psi}=1-z K_{\tau 0}-z K_{\tau m}
$$

Then, the gradient of the total electro-chemical potential is calculated as: 


$$
\begin{gathered}
\boldsymbol{\nabla} u=\frac{R T}{C} f_{\psi}\left(1+\frac{\partial \ln (\gamma)}{\partial \ln (C)}\right) \nabla C+R f_{\psi} \ln (\gamma C) \nabla T=\frac{R T}{C} f_{\psi} f_{\gamma}\left(1+\frac{C}{T f_{\gamma}} \ln (\gamma C) \frac{\partial T}{\partial C}\right) \nabla C \\
\text { with } f_{\gamma}=1+\frac{\partial \ln (\gamma)}{\partial \ln (C)}
\end{gathered}
$$

The second term in the RHS of Eq. 14 represents the coupling effect between temperature and chloride concentration on the electro-chemical potential gradient. In this study, the room temperature is $296 \mathrm{~K}, C$ is between $0 \sim 1 \mathrm{~mol} / \mathrm{L}, \gamma$ is between $0.6 \sim 1, f_{\gamma}$ is between $0.95 \sim$ 1.21 [14]. Since the maximum temperature difference considered in this research is $25 \mathrm{~K}$ and assuming that $\partial T / \partial C \approx \Delta T / \Delta C=25 \mathrm{~K} \mathrm{~L} / \mathrm{mol}$, which means that the absolute value of the coupling term is less than 0.053 which is negligible as compared to 1 . Therefore, $\nabla u$ can be simplified as:

$$
\boldsymbol{\nabla} u=\frac{R T}{C} f_{\psi} f_{\gamma} \nabla C
$$

The presented gradient of the electro-chemical potential in Eq. 15 is now related to the concentration gradient using $f_{\psi}$ which represents the effect of the overall electrical potential (see Eq. 13), and $f_{\gamma}$ which represents the effect of ionic activity, that is equal to 1 for an ideal (very dilute) solution. The activity coefficient accounts for the interactions of the different types of ions, such as ion-solvent interaction (electrophoretic effect) and ion-ion interaction (relaxation effect), that take place in a real solution [59]. As reported in literature [12], the chemical activity has a significant effect on the chemical potential and various formulations have been proposed to model it. In this work the Extended Debye-Hückel equation $[60,61]$ is used to describe the relationship between activity coefficient and concentration and it reads as

$$
\frac{\partial \ln (\gamma)}{\partial \ln (C)}=-2.302 I\left[\frac{A_{\gamma}\left|z_{+} z_{-}\right|}{2 \sqrt{I}\left(1+B_{\gamma} a^{\prime} \sqrt{I}\right)^{2}}-B^{*}\right]
$$

where $A_{\gamma}, B_{\gamma}$ are parameters related to temperature. However, experimental results show that the dependence of activity coefficient on temperature is very weak $[62,63]$. Therefore, $A_{\gamma}, B_{\gamma}$ 
are assumed to be constant in this research and the values at $25^{\circ} \mathrm{C}$ reported in [61] are adopted hereafter, i.e. $A_{\gamma}=0.509 \mathrm{dm}^{1 / 2} / \mathrm{mol}^{1 / 2}$ and $B_{\gamma}=3.286 \mathrm{dm}^{1 / 2} /\left(\mathrm{mol}^{1 / 2} \cdot \mathrm{nm}\right)$. The coefficient $a^{\prime}$ is a constant corresponding to the radius of the ion and $B^{*}$ is a model parameter. The term $I$ represents the ionic strength, $I=\left(n_{+} z_{+}{ }^{2}+n_{-} z_{-}{ }^{2}\right) C / 2$, in which $z_{+}$and $z_{-}$are charge number of cations and anions, respectively, $n_{+}$and $n_{-}$are the ion identification number of cations and anions, respectively. Thus, the effect of chemical activity of pore solution on the electro-chemical potential is given by the coefficient $f_{\gamma}$ that has the following expression

$$
f_{\gamma}=1-2.302 I\left[\frac{A_{\gamma}\left|z_{+} z_{-}\right|}{2 \sqrt{I}\left(1+B_{\gamma} a^{\prime} \sqrt{I}\right)^{2}}-B^{*}\right]
$$

\subsection{Effect of solution concentration}

Chloride diffusion coefficients of concrete determined from the conventional diffusion or migration tests are not constant but are strongly dependent on the concentration $[13,64,65,66,67$, 68]. The flux of ionic species in concentrated solution is different from a flux in dilute solution because ions are very close to each other and that creates mechanical interactions or frictional effects between the moving ions. As the concentration increases, these effects become more important to consider. For a concentrated electrolytic solution the multi-component diffusion equation can be expressed as [13, 69]

$$
C_{i} \boldsymbol{\nabla} u_{i}=\sum_{j} K_{i j}\left(v_{j}-v_{i}\right)=R T \sum_{j} \frac{C_{i} C_{j}}{\left(\sum_{i} C_{i}\right) \mathscr{D}_{i j}}\left(v_{j}-v_{i}\right)
$$

where $u_{i}$ is the electro-chemical potential of species $i, K_{i j}$ is friction coefficient of species $i$ with respect to the species $j, v_{i}$ is the average velocity of species $i, \mathscr{D}_{i j}$ is a diffusion coefficient describing the interaction between the $i$ and $j$ species. Again, while this potential can be theoretically represented, concrete pore solution is rich in multiple ionic species and their concentrations have to be explicitly monitored so that the different ionic diffusion parameters can be calibrated. Thus as a reasonable simplification, a binary solution is only considered here. For a binary electrolytic solution composed of anions, cations, and solvent, the Eq. 18 can be written for anions (ions with negative charge) as 


$$
\begin{aligned}
C_{-} \nabla u_{-} & =K_{0-}\left(v_{0}-v_{-}\right)+K_{+-}\left(v_{+}-v_{-}\right)= \\
& =R T \frac{C_{-} C_{+}}{\left(C_{-}+C_{+}+C_{0}\right) \mathscr{D}_{-+}}\left(v_{+}-v_{-}\right)+R T \frac{C_{-} C_{0}}{\left(C_{-}+C_{+}+C_{0}\right) \mathscr{D}_{-0}}\left(v_{0}-v_{-}\right)
\end{aligned}
$$

Here the subscript indices "-,," +" and "0" are used to refer to anions, cations and solvent respectively. So $v_{-}, v_{+}$and $v_{0}$ are the velocities and $C_{-}, C_{+}$and $C_{0}$ are the concentration of the anions, cations and solvent, respectively, $\mathscr{D}_{-0}$ represents the diffusion coefficient of the anion in dilute solution. Setting $\beta_{v}=v_{+} / v_{-}$and $f=\mathscr{D}_{-0} / \mathscr{D}_{-+}$, considering a neutral $\mathrm{NaCl}$ salt solution, i.e. $C_{-}=C_{+}=C, v_{-}$being the velocity of chloride ions $v_{C l}$, and $\mathscr{D}_{-0}$ being the diffusion coefficient of chloride in dilute solution $D_{0}$ and also considering fully saturated situation $\left(v_{0}=0\right)$, the Eq. 19 can be simplified as

$$
C \nabla u=-R T \frac{C f\left(1-\beta_{v}\right)+C_{0}}{\left(2 C+C_{0}\right) D_{0}}\left(C v_{C l}\right)
$$

Since $J_{s}=C v_{C l}$ by definition, then Eq .20 can be rearranged as

$$
J_{s}=-f_{s} \frac{D_{0}}{R T} C \nabla u \text { with } f_{s}=\frac{2 C+C_{0}}{C f_{v}+C_{0}} \text { and } f_{v}=f\left(1-\beta_{v}\right)
$$

where $f_{s}$ represents the effect of solution concentration and $f_{v}$ is the friction coefficient which is usually much greater than 2 [14]. Substituting Eq. 15 into Eq. 21 one gets the relation between the flux in the concentrated pore solution and the concentration gradient as

$$
J_{s}=-f_{\psi} f_{\gamma} f_{s} D_{0} \nabla C=-D_{s} \nabla C, \text { with } D_{s}=f_{\gamma} f_{\psi} f_{s} D_{0}
$$

\subsection{Effect of age-dependent pore structure}

The overall diffusion coefficient of chloride, $D_{c}$, in a cementitious material is also governed by porosity and pore characteristics such as shape, size, orientation, spatial distribution, connectivity, and size variation [55]. Moreover, the pore structure is not constant but changes as the material ages. To consider these features, the diffusion of chloride in the pore solution of porous 
materials can be expressed as $D_{c}=f_{\phi} D_{s}$, which implies that the flux of chloride in a cementitous material can be formulated as $J_{c}=\left(J_{0} f_{s} f_{\gamma} f_{\psi}\right) f_{\phi}$, in which $f_{\phi}$ is a coefficient that accounts for the porosity and its characteristics $[5,70]$ as

$$
f_{\phi}=\frac{w_{c a p}}{\rho_{w}} \frac{\delta}{\tau^{2}} q(\phi)=\frac{w_{c a p}}{\rho_{w}} \tau_{a} q(\phi)
$$

As shown in Fig. 1a, $\tau$ is the tortuosity factor which is defined as the ratio of the effective length, $L_{e f f}$, which is the shortest path that connect two points in the pore structure, to the length of the straight line that connects the two points [55]; $\delta$ is the constrictivity factor which represents a measure of the presence of bottlenecks in the pores path by relating the minimum pore diameter to the maximum pore diameter. If the cross section of pores is constant, then the constrictivity factor, $\delta$ is equal to 1 . If the pore structure becomes narrow at some point, then $0<\delta<1$ or if the pores are not connected, then $\delta=0$ [70], see Fig.1b. The coefficient $\tau_{a}=\delta / \tau^{2}$ is the apparent tortuosity factor and is assumed to be a free model parameter which lumps together the effect of tortuosity and constrictivity since it is extremely difficult to identify separately the effect of $\delta$ and

$\tau$. In addition to that the pore volume has its own effect which is accounted for by introducing the function $q(\phi)$ based on Kozeny-Carman model [71] as

$$
q(\phi)=\left(\frac{\phi}{\phi_{0}}\right)^{3}\left(\frac{1-\phi_{0}}{1-\phi}\right)^{2}
$$

where $\phi$ is the current porosity that changes over time and $\phi_{0}$ is the initial porosity at the initial setting of the material. The effect of porosity is very important when trying to use accelerated lab experiments to predict long term behavior because concrete continues to hydrate and its pore structure densifies and becomes more tortuous. 

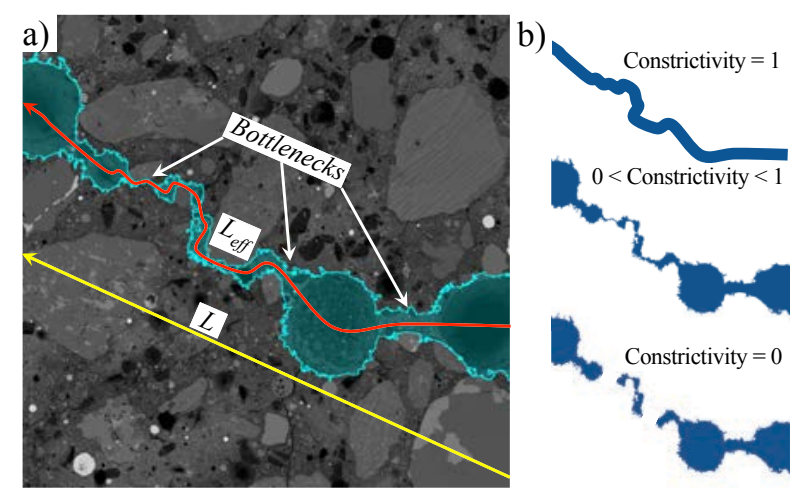

Figure 1: Concrete porous microstructure showing a) tortuous path through pores, and b) different constrictivity conditions

As concrete ages, its internal structure changes due to many chemical and physical reactions that are also influenced by the curing conditions. In the presented model, the evolution of porosity and cement hydration is also obtained by employing the HTC model that, once the mix composition is given, provides the hydration degree $\alpha_{c}$, capillary water $w_{\text {cap }}$ and non-evaporable water $w_{n}$ at a specific time. The porosity can be calculated using capillary water content at full saturation as $\phi=w_{c a p}^{\text {sat }} / \rho_{w}$, in which $w_{c a p}^{\text {sat }}$ is calculated with $h=1$.

\subsection{Effect of temperature}

It is well known from thermodynamics that temperature plays a significant role in the diffusion process. Consequently, the diffusivity of ionic species in pure water varies under different temperatures. For this reason, the diffusivity is modified as $f_{T} D_{0}$ to consider the effect of variable temperature, in which $D_{0}$ is determined at the reference temperature $T_{0}$ and $f_{T}$ is a factor expressed as

$$
f_{T}=\exp \left[\frac{U}{R}\left(\frac{1}{T_{0}}-\frac{1}{T}\right)\right]
$$

where $U$ is the activation energy of the diffusion process.

Considering the effect of temperature, the diffusion flux of chloride ions and its divergence in a cementitious material in saturated conditions (where the fluid mass is stationary $v=0$ ) can be written as 


$$
J_{c}=f_{\phi} f_{\gamma} f_{\psi} f_{s} f_{T} J_{0} \quad \text { and } \quad \boldsymbol{\nabla} \cdot J_{c}=-f_{\phi} f_{\gamma} f_{\psi} f_{s} f_{T} D_{0} \boldsymbol{\nabla}^{2} C=-D_{c} \boldsymbol{\nabla}^{2} C
$$

where $D_{c}$ is the diffusivity coefficient of chloride in concrete, $D_{c}=f_{s} f_{\phi} f_{\gamma} f_{\psi} f_{T} D_{0}$. Combining Eqs. 2,8 , and 26 , the transport of chloride in saturated conditions $(\partial h / \partial t=0)$ is governed by the following equation

$$
\left(\frac{w_{c a p}}{\rho_{w}}+\frac{w_{s} A_{0} C_{b}}{C}\right) \frac{\partial C}{\partial t}+\frac{w_{s} U_{b} C_{b}}{R T^{2}} \frac{\partial T}{\partial t}=D_{c} \nabla^{2} C-\frac{C \dot{\alpha}_{c}}{\rho_{w}} \frac{\partial w_{c a p}}{\partial \alpha_{c}}-\left(c \dot{\alpha}_{c}+\dot{w}_{n}\right) \frac{C_{b}}{\rho_{s}}
$$

For unsaturated condition the advection term must be considered in $J_{c}$ (see Eq. 9). The fluid mass velocity, $v$, can be expressed as $v=-J_{w} / \rho_{w}$ where $J_{w}$ is the flux of water mass per unit volume. Following Di Luzio and Cusatis [38], $J_{w}$ is proportional to the spatial gradient of $h$, thus one can write $J_{w}=-D_{h} \nabla h$, in which $D_{h}$ is the moisture permeability that is a nonlinear function of $T$ and $h$ [38]. This gives $v=-D_{h} \nabla h / \rho_{w}$ and consequently $J_{c}=-D_{c} \nabla C-C D_{h} \nabla h / \rho_{w}$. Then the previous Eq. 27 can be generalized for unsaturated conditions as

$$
\begin{aligned}
\left(\frac{w_{c a p}}{\rho_{w}}+\frac{w_{s} A_{0} C_{b}}{C}\right) \frac{\partial C}{\partial t}+\frac{w_{s} U_{b} C_{b}}{R T^{2}} \frac{\partial T}{\partial t}+\frac{C}{\rho_{w}} \frac{\partial w_{c a p}}{\partial h} \frac{\partial h}{\partial t}= \\
D_{c} \nabla^{2} C+C \frac{D_{h}}{\rho_{w}} \nabla^{2} h-\frac{C \dot{\alpha}_{c}}{\rho_{w}} \frac{\partial w_{c a p}}{\partial \alpha_{c}}-\left(c \dot{\alpha}_{c}+\dot{w}_{n}\right) \frac{C_{b}}{\rho_{s}}
\end{aligned}
$$

\section{Coupling with the HTC model}

The spacial and temporal distributions of temperature, $T$, relative humidity, $h$, and the hydration degree, $\alpha_{c}$, are needed for the chloride diffusion model to define both environmental effects and concrete aging. These fields are obtained by employing the Hygro-Thermo-Chemical (HTC) model by Di Luzio and Cusatis [38, 39], which simulates the coupled heat and moisture transport with a good prediction of cement hydration process and its effects on generating heat sources and consuming moisture. Following $[38,39]$ the moisture mass and enthalpy balance equations read:

$$
\boldsymbol{\nabla} \cdot\left(D_{h} \boldsymbol{\nabla} h\right)-\frac{\partial w_{e}}{\partial h} \frac{\partial h}{\partial t}-\frac{\partial w_{e}}{\partial \alpha_{c}} \dot{\alpha}_{c}-\dot{w}_{n}=0
$$




$$
\boldsymbol{\nabla} \cdot\left(\lambda_{t} \boldsymbol{\nabla} T\right)-\rho c_{t} \frac{\partial T}{\partial t}+\dot{\alpha}_{c} c \tilde{Q}_{c}^{\infty}=0
$$

where $D_{h}$ is moisture permeability, $w_{e}$ is evaporable water, $\dot{w}_{n}=0.253 \dot{\alpha}_{c} c$ is rate of nonevaporable water, $\rho$ is the mass density of concrete, $c_{t}$ is the isobaric heat capacity (specific heat), $\lambda_{t}$ is the heat conductivity, $\tilde{Q}_{c}^{\infty} \approx 450 \mathrm{~kJ} / \mathrm{kg}$ is the hydration enthalpy.

The moisture permeability is dependent on relative humidity $h$ and temperature $T$, as

$$
D_{h}(h, T)=\exp \left(\frac{E_{a d}}{R T_{0}}-\frac{E_{a d}}{R T}\right) D_{h 1}\left[1+\left(\frac{D_{h 1}}{D_{h 0}}-1\right)(1-h)^{n}\right]^{-1}
$$

where $T_{0}=296 \mathrm{~K}, E_{a d} / R \approx 2700 \mathrm{~K}[38,39], D_{h 0}$ and $D_{h 1}$ represent moisture permeability for a completely dry situation $(h=0)$ and completely saturated situation $(h=1)$. The evaporable water content (the so-called sorption isotherm) can be assumed to be a function of relative humidity and degree of hydration $[54,38]$ as

$$
w_{e}\left(h, \alpha_{c}\right)=k_{v g}^{c} \alpha_{c} c\left[1-\frac{1}{e^{10\left(g_{1} \alpha_{c}^{\infty}-\alpha_{c}\right) h}}\right]+K_{1}\left(\alpha_{c}\right)\left[e^{10\left(g_{1} \alpha_{c}^{\infty}-\alpha_{c}\right) h}-1\right]
$$

where the first term represents the gel water (physically bound) whereas the second term represents the capillary water $w_{c a p}$, and $\alpha_{c}^{\infty}$ is the asymptotic degree of hydration. The coefficient $K_{1}\left(\alpha_{c}\right)$ is given by

$$
K_{1}\left(\alpha_{c}\right)=\frac{w_{0}-0.188 \alpha_{c} c-k_{v g}^{c} \alpha_{c} c\left[1-e^{-10\left(g_{1} \alpha_{c}^{\infty}-\alpha_{c}\right)}\right]}{e^{10\left(g_{1} \alpha_{c}^{\infty}-\alpha_{c}\right)}-1}
$$

The material parameters are $g_{1}$ and $k_{v g}^{c}$ to be calibrated from experimental data or from multiscale analysis [72]. The moisture capacity is given by $\partial w_{e} / \partial h$ (derivative of the sorption isotherm to respect to $h$ that is needed in the first equation in Eq. 29.

The hydration degree, $\alpha_{c}$, that represents the fraction of Portland clinker fully reacted with water is used to characterize cement hydration $[38,73,74,75]$ and its evolution is expressed as

$$
\dot{\alpha}_{c}=\frac{A_{c 1} e^{-\eta_{c} \alpha_{c} / \alpha_{c}^{\infty}} e^{-E_{a c} / R\left(T-T_{0}\right)}}{1+(5.5-5.5 h)^{4}}\left(\frac{A_{c 2}}{\alpha_{c}^{\infty}}+\alpha_{c}\right)\left(\alpha_{c}^{\infty}-\alpha_{c}\right)
$$


where $E_{a c} / R \approx 5000 \mathrm{~K}, T_{0}=296 \mathrm{~K}, \eta_{c}, A_{c 1}, A_{c 2}$ are material parameters to be calibrated from experimental data or from multi-scale analysis [72]. For details on the calibration and validation of this theory see [39]. This model was coupled in many research works with LDPM to successfully represent and predict concrete long term behavior under coupled shrinkage, creep, ASR degradation $[76,77,34,35,36,78,79,32,33]$.

\section{Numerical implementation}

\section{1. mesh generation}

The proposed model for chloride transport in cementitious materials is implemented into a discrete conduit network generated from the meso-scale geometry of the Lattice Discrete Particle Modeling (LDPM) $[80,81]$ that constructs the discrete mesh considering the geometrical characterization of concrete heterogeneity. As shown in Fig. 2, the coarse aggregates in concrete are assumed to be spherical and then placed through try-and-reject random procedure into the volume. Based on the Delaunay tetrahedralization from the centers of the considered aggregates a system of polyhedral cells (see Fig. 2) is created using a tessellation procedure. The surfaces between polyhedral cells are located where the cement paste is likely to be and where the diffusion/transport phenomena take place. 

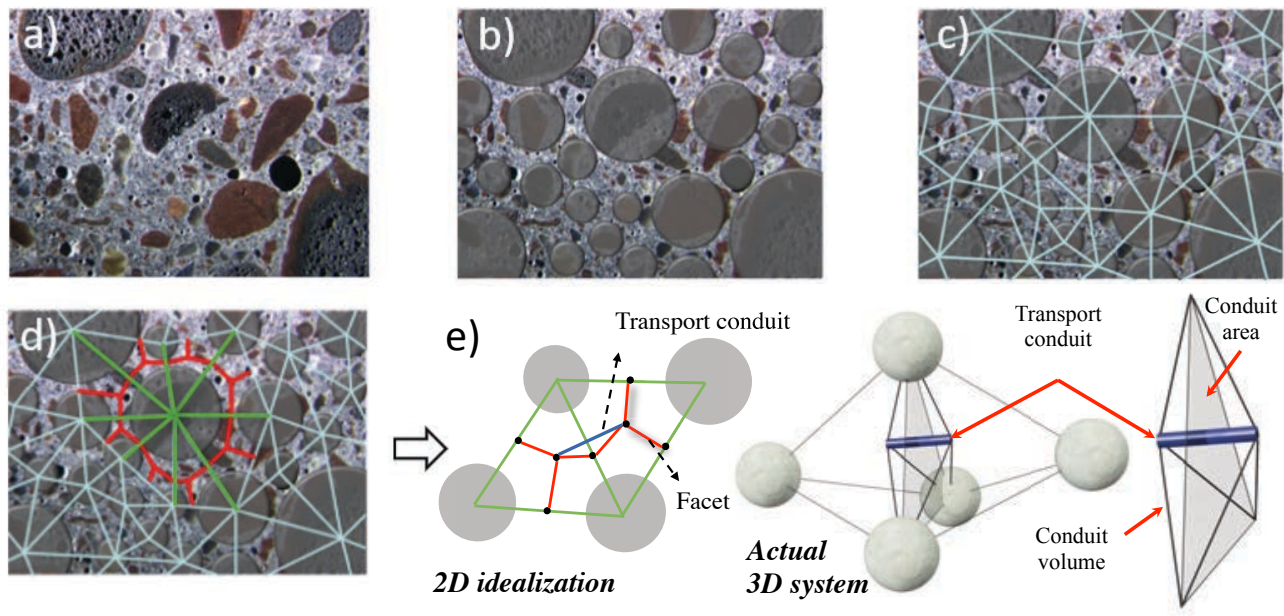

Figure 2: Mesh generation: (a) Concrete material;b) Supporting particles for grain generation; (c) Delaunay tetrahedralization (d) Tessellation (e) 2D idealization and 3D visualization of the conduit element connecting the centers of two tetrahedrons

These preferential diffusion paths are identified by the interconnected tetrahedrons (triangles in 2D) that create the transport lattice network, whose nodes coincide with the tessellation points and their connection generates the 1D transport conduits, see Fig. 2e. This network of 1D transport conduits previously presented by [82], is adopted here to simulate the transport of heat, moisture and chloride ions.

Compared with standard continuous finite element approaches, the advantage of the discrete diffusion network is that it can simulate the transport process anchored in the heterogeneous meso-scale structure of the material leading to transport paths of chloride ions that are neither straightforward nor homogeneous even at the same depth from the side exposed to the chloride solution. Thus the model can capture naturally the tortuosity at the meso-scale that is generated by aggregate grain size distribution. However, the tortuosity at finer scales is then captured by the apparent tortuosity parameter $\tau_{a}$ introduced earlier in Sec. 3.4. Following our main idea of keeping the formulation as simple as possible yet capturing as much physics with available experimental data, in this study, due to the lack of information about the percentage concentrations of chlorides within the matrix and aggregate, the conduits represent their volume-averaged properties. Nevertheless, the modeling framework is already capable of representing the more 
complex and realistic conditions if experimental data become available.

The computational cost of the proposed diffusion network on a single processor is proportional to the number of adopted particles due to the explicit integration scheme. For example, considering a cylinder of $200 \mathrm{~mm}$ high, $75 \mathrm{~mm}$ in radius, a discretization with 1934 particles (coarse aggregate pieces) ends up with 30468 nodes, while a finer discretization of the same geometry containing 4,549 particles will require 70,089 diffusion nodes. Consequently, the computational process becomes very time-consuming when a large number of particles are present. This means that the particle size distribution should be carefully selected so as to achieve a balance between computational cost and the geometrical representation of the meso-structure. The particles size distribution depends on three parameters, maximum particle size, fuller coefficient, and minimum particle size. The first can be obtained directly from the mix design and the second can be identified by fitting the concrete sieve curve. As for the last one, although a small minimum particle size characterizes better the heterogeneity of the material, in the meanwhile it leads to expensive computational cost. Hence in this study, as specified later in each studied case, the minimum particle size is chosen as small as possible while keeping the computational cost reasonable.

\subsection{Numerical solution}

To clarify the numerical solution of the proposed model it is worth rewriting the governing differential equation system for 1D case in the following general form

$$
\begin{aligned}
& a_{11} \frac{\partial h}{\partial t}+a_{12} \frac{\partial T}{\partial t}+a_{13} \frac{\partial C}{\partial t}=\frac{\partial}{\partial x}\left(d_{11} \frac{\partial h}{\partial x}\right)+\frac{\partial}{\partial x}\left(d_{12} \frac{\partial T}{\partial x}\right)+\frac{\partial}{\partial x}\left(d_{13} \frac{\partial C}{\partial x}\right)+\kappa_{1} \\
& a_{21} \frac{\partial h}{\partial t}+a_{22} \frac{\partial T}{\partial t}+a_{23} \frac{\partial C}{\partial t}=\frac{\partial}{\partial x}\left(d_{21} \frac{\partial h}{\partial x}\right)+\frac{\partial}{\partial x}\left(d_{22} \frac{\partial T}{\partial x}\right)+\frac{\partial}{\partial x}\left(d_{23} \frac{\partial C}{\partial x}\right)+\kappa_{2} \\
& a_{31} \frac{\partial h}{\partial t}+a_{32} \frac{\partial T}{\partial t}+a_{33} \frac{\partial C}{\partial t}=\frac{\partial}{\partial x}\left(d_{31} \frac{\partial h}{\partial x}\right)+\frac{\partial}{\partial x}\left(d_{32} \frac{\partial T}{\partial x}\right)+\frac{\partial}{\partial x}\left(d_{33} \frac{\partial C}{\partial x}\right)+\kappa_{3}
\end{aligned}
$$

Since the governing equations presented in the proposed model are not fully coupled, some of the coefficients in the previous Eq. 35 are null, i.e. $a_{12}=a_{13}=a_{21}=a_{23}=0$ and $d_{12}=$ $d_{13}=d_{21}=d_{23}=d_{32}=0$. The expressions of the other coefficients are $a_{11}=\partial w_{e} / \partial h, a_{22}=\rho c_{t}$, $a_{31}=C / \rho_{w}\left(\partial w_{c a p} / \partial h\right), a_{32}=w_{s} U_{b} C_{b} / R / T^{2}, a_{33}=w_{c a p} / \rho_{w}+w_{s} A_{0} C_{b} / C, d_{11}=D_{h}, d_{22}=\lambda_{t}$, 
${ }_{470} d_{31}=C D_{h} / \rho_{w}, d_{33}=D_{c}, \kappa_{1}=-\left(\partial w_{e} / \partial \alpha_{c}\right) \dot{\alpha}_{c}-\dot{w}_{n}, \kappa_{2}=\dot{\alpha}_{c} C \tilde{Q}_{c}^{\infty}, \kappa_{3}=-C \dot{\alpha}_{c} / \rho_{w}\left(\partial w_{c a p} / \partial \alpha_{c}\right)-$

$471\left(c \dot{\alpha}_{c}+\dot{w}_{n}\right) C_{b} / \rho_{s}$. Considering a 1D transport conduit, using the classical Galerkin method to 472 obtain the weak formulation of the differential problem and a finite element approximation of 473 the primary variables, $T(x, t) \cong \Sigma_{i}^{N} T_{i}(t) N_{i}(x), h(x, t) \cong \Sigma_{i}^{N} h_{i}(t) N_{i}(x)$, and $C(x, t) \cong \Sigma_{i}^{N} C_{i}(t) N_{i}(x)$ ${ }_{474}\left(N_{i}(x)\right.$ are linear 1D shape functions), and assembling the total element level matrices, one can 475 obtain the global equation as following

$$
\mathbf{M u}+\mathbf{K u}=\mathbf{F}
$$

476

477

478 matrices given by

$$
\left.\begin{array}{r}
\mathbf{M}^{e l}=\frac{A_{e} l_{e}}{6 \delta}\left[\begin{array}{cccccc}
2 a_{11} & 0 & 0 & -a_{11} & 0 & 0 \\
0 & 2 a_{22} & 0 & 0 & -a_{22} & 0 \\
2 a_{31} & 2 a_{32} & 2 a_{33} & -a_{31} & -a_{32} & -a_{33} \\
-a_{11} & 0 & 0 & 2 a_{11} & 0 & 0 \\
0 & -a_{22} & 0 & 0 & 2 a_{22} & 0 \\
-a_{31} & -a_{32} & -a_{33} & 2 a_{31} & 2 a_{32} & 2 a_{33}
\end{array}\right] \\
\mathbf{K}^{e l}=\frac{A_{e}}{l_{e}}\left[\begin{array}{cccccc}
d_{11} & 0 & 0 & -d_{11} & 0 & 0 \\
0 & d_{22} & 0 & 0 & -d_{22} & 0 \\
d_{31} & 0 & d_{33} & -d_{31} & 0 & -d_{33} \\
-d_{11} & 0 & 0 & d_{11} & 0 & 0 \\
0 & -d_{22} & 0 & 0 & d_{22} & 0 \\
-d_{31} & 0 & -d_{33} & d_{31} & 0 & d_{33}
\end{array}\right] \\
\mathbf{F}^{e l}=\frac{A_{e} l_{e}}{2 \delta}\left[\kappa_{1} \kappa_{2} \kappa_{3} \kappa_{1} \kappa_{2} \kappa_{3}\right.
\end{array}\right]^{T} \quad
$$

479 where $l_{e}$ is the conduit length, $A_{e}$ is the conduit area at the interface between the two interacting 
tetrahedrons (projection of the shaded area along the direction of $l_{e}$ ). Since the conduit represents a volume defined by double pyramids (see Fig. 2e), $\delta=3$ is used to represent the correct element volume.

Temporal integration of Eq. 36 is performed by applying Crank-Nicolson method (a central difference method) which has been widely used as a numerical solution for partial differential equations due to its unconditional stability (for constant coefficients). For each time step, from $t_{n}$ to $t_{n+1}=t_{n}+\Delta t$, Eq. 36 is solved using the Crank-Nicholson method as

$$
\left(\mathbf{M}_{n+1 / 2}-\frac{1}{2} \Delta t \mathbf{K}_{n+1 / 2}\right) \mathbf{u}_{n+1}^{i}=\left(\mathbf{M}_{n+1 / 2}+\frac{1}{2} \Delta t \mathbf{K}_{n+1 / 2}\right) \mathbf{u}_{n}+\Delta t \mathbf{F}_{n+1 / 2}
$$

This calculation is repeated updating the coefficients, $\mathbf{M}_{n+1 / 2}, \mathbf{K}_{n+1 / 2}$, and $\mathbf{F}_{n+1 / 2}$ using the mid time-step value, $\left(\mathbf{u}_{n+1}^{i+1}+\mathbf{u}_{n+1}^{i}\right) / 2$, until the convergence is achieved, i.e. the Euclidean norm of the difference of nodal values between two iterations is satisfactorily small, $\left\|\mathbf{u}_{n+1}^{i+1}-\mathbf{u}_{n+1}^{i}\right\|<10^{-4}$.

Finally, the numerical calculation of the proposed formulation requires, at each discrete element, the time integration of the internal variables, such as the hydration degree. Expressing an internal variable as $y$ the time-dependent evolution law can be generally written as $\dot{y}=$ $f(y, h, T, C)$. It can be effectively integrated using the second order Runge-Kutta formula (midpoint method), in this way the value of the internal variable $y_{n+1}$ at time $t_{n+1}$ is evaluated as $y_{n+1} \approx y_{n}+\Delta t_{n} f\left(y_{n+1 / 2}, h_{n+1 / 2}, T_{n+1 / 2}, C_{n+1 / 2}\right)$, in which $y_{n}$ is the value of the internal variable at time $t_{n}, \Delta t_{n}$ is the time increment, $y_{n+1 / 2}=\left(y_{n+1}+y_{n}\right) / 2, h_{n+1 / 2}=\left(h_{n+1}+h_{n}\right) / 2$, and $T_{n+1 / 2}=\left(T_{n+1}+T_{n}\right) / 2$.

The proposed formulation is implemented in Mars, a multi-purpose computational software for the explicit dynamic simulation of structural performance [83].

\section{Identification of model parameters}

As explained before, the model accounts for multiple physical phenomena yet, it uses literature published data regarding many of the physical quantities involved in representing such phenomena. Note that the chloride diffusion model also depends on the HTC model. To summarize all these model parameters (both the chloride diffusion and the HTC model parameters), 
Table 1 enlists each group of parameters that are used to represent a specific phenomenon. In the table, values and sources are listed for all independently measured or reasonably assumed parameters.

Table 1: Known or independently identified parameters used in different modeled phenomena

\begin{tabular}{|c|c|c|c|c|}
\hline Modeled Phenomena & Parameter (unit) & Value [source] & Parameter (unit) & Value [source] \\
\hline \multirow[t]{2}{*}{ Cement hydration } & $E_{a c} / R(\mathrm{~K})$ & $5000[74]$ & $A_{c 1}\left(\mathrm{~h}^{-1}\right)$ & $1.50 \times 10^{7}[39]$ \\
\hline & $A_{c 2}(-)$ & $1.0 \times 10^{-4}[39]$ & $\eta_{c}(-)$ & $5.5[39]$ \\
\hline Sorption isotherm & $k_{v g}^{c}(-)$ & 0.2 [39] & $g_{1}(-)$ & 1.25 [39] \\
\hline \multirow{3}{*}{$\begin{array}{l}\text { Moisture diffusion } \\
\text { and heat transfer }\end{array}$} & $E_{a d} / R(\mathrm{~K})$ & $2700[84]$ & $\lambda_{t}\left(\mathrm{~W} / \mathrm{m}{ }^{\circ} \mathrm{C}\right)$ & $2.3[85]$ \\
\hline & $c_{t}(\mathrm{~J} / \mathrm{kg} / \mathrm{K})$ & $1100[86]$ & $Q_{c}^{\infty}(\mathrm{kJ} / \mathrm{kg})$ & $450[36]$ \\
\hline & $T_{0}(\mathrm{~K})$ & 296 [defined] & & \\
\hline \multirow{2}{*}{$\begin{array}{l}\text { Concrete compo- } \\
\text { nents densities }\end{array}$} & $\rho\left(\mathrm{kg} / \mathrm{m}^{3}\right)$ & $2400[86]$ & $\rho_{c}\left(\mathrm{~kg} / \mathrm{m}^{3}\right)$ & $1800[86]$ \\
\hline & $\rho_{s}\left(\mathrm{~kg} / \mathrm{m}^{3}\right)$ & 2340 [87] & & \\
\hline \multirow{2}{*}{$\begin{array}{l}\text { Chloride chemical } \\
\text { activity }\end{array}$} & $A_{\gamma}\left(\mathrm{dm}^{0.5} / \mathrm{mol}^{0.5}\right)$ & $0.509[61]$ & $a^{\prime}(\mathrm{nm})$ & $0.4[61]$ \\
\hline & $\begin{array}{l}B_{\gamma}\left(\mathrm{dm}^{0.5} /\left(\mathrm{mol}^{0.5} .\right.\right. \\
\mathrm{nm}))\end{array}$ & $3.286[61]$ & $B^{*}(\mathrm{dm} / \mathrm{mol})$ & $0.045[61]$ \\
\hline \multirow{2}{*}{$\begin{array}{l}\text { Pore solution } \\
\text { chemistry }\end{array}$} & $K_{\tau 0}(-)$ & $-0.207[14]$ & $K_{\tau \mathrm{m}}(-)$ & $-0.402[14]$ \\
\hline & $D_{0}\left(\mathrm{~m}^{2} / \mathrm{s}\right)$ & $2.03 \times 10^{-9}[14]$ & $C_{0}\left(\mathrm{~mol} / \mathrm{m}^{3}\right)$ & $\begin{array}{l}55000 \quad \text { [calcu- } \\
\text { lated] }\end{array}$ \\
\hline \multirow{2}{*}{$\begin{array}{l}\text { Charge and ion } \\
\text { identification }\end{array}$} & $z_{+}$ & $+1[60]$ & $z_{-}$ & $-1[60]$ \\
\hline & $n_{+}$ & $1[60]$ & $n_{-}$ & $1[60]$ \\
\hline
\end{tabular}

The rest of the model parameters need to be calibrated using specific experimental data relevant to them. Typically, there are two sets of parameters to be calibrated. The first set is relevant to the HTC model and is based on the concrete mix design, curing conditions and ageing. Since the chloride penetration model depends on $\alpha_{c}, h$, and $T$ to capture various microstructural effects as well as thermal and hygral effects, HTC model parameters must be determined first so that their calibration process does not affect the robustness of the calibration of the chloride diffusion parameters. This condition is followed in all calibrations performed in this work. In some cases 
though, chloride penetration tests are performed on samples that have already reach a significant degree of hydration (old enough) and the tests are performed under isothermal and fully saturated conditions. For such cases, the pore structure characteristics are fixed and no heat or moisture sources/sinks are present. As a result, the pore structure effect is not changing. This means that even if not enough data is present about the exact composition of the material, chloride penetration parameters can be still calibrated after choosing appropriate values of the HTC parameters from literature. In this case, one must notice that the identified parameter values will be sensitive to the difference between assumed HTC parameters and the ones representing the actual material. Yet, this is an acceptable approximation if experimental data to evaluate the hydration degree and other HTC related phenomena are lacking. The detailed procedure regarding the calibration of HTC parameters and the needed experiments to perform the calibration are described in [39] to which the reader is directed for additional information.

Regarding the proposed chloride penetration model, the presented formulation has only four basic free parameters under isothermal conditions that need to be identified from experimental results: apparent tortuosity factor $\tau_{a}$, the friction coefficient $f_{v}$, and two material parameters related to binding capacity, $A_{0}$ and $B_{0}$. Typically, all parameters can be calibrated using chloride penetration profiles data. The apparent tortuosity factor $\tau_{a}$ plays an important role in the chloride penetration depth. Parameters $A_{0}$ and $B_{0}$ determine the binding capacity and can be identified by fitting the bound or total chloride concentration at the boundary, where the free chloride concentration is always equal to the chloride concentration in the external solution. The friction coefficient $f_{v}$ represents the concentration dependence of chloride diffusion, and it influences the curvature of the concentration-depth profile. Therefore, ideal identification of the four parameters can be achieved using two chloride concentration-depth profiles relevant to two different boundary concentrations. First, $A_{0}$ and $B_{0}$ are identified by matching the chloride concentration at the surface, then $\tau_{a}$ is calibrated by matching the chloride penetration depth, and finally $f_{v}$ is calibrated by fitting the curvature. Of course, due to experimental data scatter and the slight dependence of the concentration-depth profile on each of the parameters, fine tuning is typically inevitable but based on the calibrations performed in this work, fine tuning is very minimal if 
Table 2: Chloride diffusion model calibrated free parameters

\begin{tabular}{|c|c|c|c|c|c|}
\hline Parameter & Symbol (unit) & Sec. 7.1 & Sec. 7.2 & Sec. 7.3 & Sec. 7.4 \\
\hline Apparent tortuosity & $\tau_{a}(-)$ & 0.054 & 0.11 & 0.4 & 0.06 \\
\hline Material parameter for chloride binding & $A_{0}(-)$ & 0.45 & 0.55 & 0.7 & 0.5 \\
\hline Material parameter for chloride binding & $B_{0}(-)$ & 1.0 & 1.46 & 1.9 & 1.25 \\
\hline Friction coefficient & $f_{v}(-)$ & 400 & 600 & 600 & 500 \\
\hline Activation energy for chloride diffusion & $U(\mathrm{~J} / \mathrm{mol})$ & - & 22450 & 40000 & - \\
\hline Activation energy for chloride binding & $U_{b}(\mathrm{~J} / \mathrm{mol})$ & - & 5810 & 33200 & - \\
\hline
\end{tabular}

two experimental curves are used. Note that all of these four parameters have to be identified at the same reference temperature $T_{0}$ (typically room temperature).

To consider the effect of temperature variation, two additional parameters need to be calibrated by fitting an additional chloride concentration-depth profile at a different temperature $T$ : the activation energy of the diffusion process $U$ and the activation energy for the binding process $U_{b}$. The parameter $U$ considers the effect of temperature on the diffusivity, so it can be calibrated by matching the change in maximum penetration depth. $U_{b}$ considers the effect of temperature on binding capacity, so it can be calibrated by matching the bound or total concentration at the boundary. Thus, for cases with thermal changes, a minimum of three chloride concentrationdepth profiles are needed, two of them relevant to two different boundary concentrations and one of them is at the same boundary concentration of either of the first two but at a different temperature.

It is important to say that the aforementioned calibration procedure represents an ideal case in which, all the needed experimental data are available. For cases where parts of the data are not available, or if data is very scattered, one can slightly deviate from this procedure yet all efforts have to be made to follow it as closely as possible.

\section{Numerical simulations of experimental literature}

The three-dimensional diffusion network, shown in Fig. 2e and presented in the previous section, is hereafter used to simulate chlorides diffusion in cementitious materials. The capability of the proposed model is verified through the numerical simulations on both cement paste and concrete. The numerical simulations are performed with reference to four experimental data sets 
taken from the literature. The data were chosen to represent simple to complex cases, starting with tests on cement paste at constant [88] and different temperatures [22], then tests on concrete samples with different temperature and chloride concentration combinations [23]. These three different tests cover all model functionality under fully saturated conditions. The last set of experiments is relevant to testing chloride penetration in partially saturated concrete [24] which attests to effectiveness of the model since it does not postulate any phenomenological assumptions to account for non-saturated flow conditions and it just covers it through the coupling between the different physical processes.

\subsection{Diffusion in saturated cement paste: Effect of exposure duration}

The first experimental investigation considered in this study is reported in [88] in which they adopted X-ray scanning to measure iodide ions instead of chloride ions to quantify the chloride transport, based on the fact that although iodide and chloride ions have similar penetration depth the latter is much more convenient to detect. With a mixture proportion that comprised $850 \mathrm{~g}$ cement (US ASTM C150 Type I) and $340 \mathrm{~g}$ water, samples were cast and cured for 35 days immersed in water. All samples are $9.5 \mathrm{~mm}$ in diameter and $30 \mathrm{~mm}$ in height.

Later the samples were sealed at room temperature $\left(23^{\circ} \mathrm{C}\right)$ for 50 days. Following that, the top end of the sample was covered with $0.6 \mathrm{~mol} / \mathrm{L}$ potassium iodide solution up to 14 days. The curing conditions and the experimental procedure are accurately modelled in the numerical analyses. 


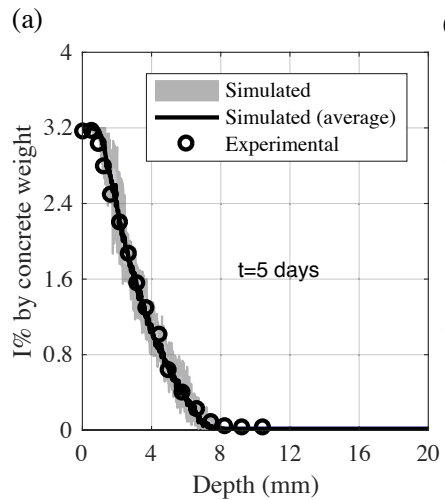

(b)

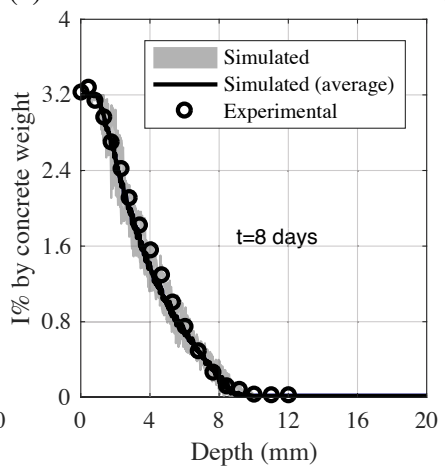

(c)

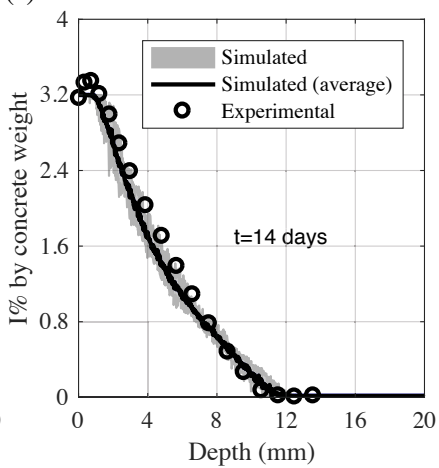

Figure 3: Experimental [88] and numerical results of iodine penetration for different ponding durations: a) 5 days; b) 8 days; c) 14 days

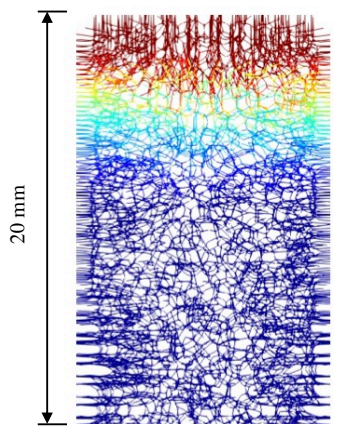

$\mathrm{t}=5$ days

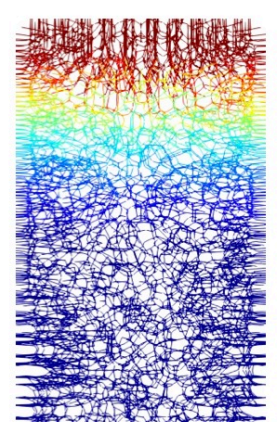

$\mathrm{t}=8$ days

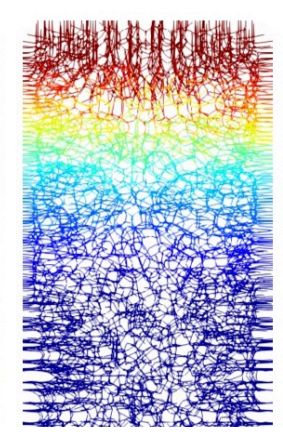

$\mathrm{t}=14$ days

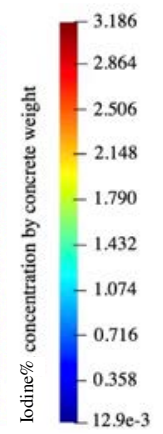

Figure 4: Contour of iodine concentration for different ponding durations

The test results after 14-day ponding are used for calibration (see Fig. 3c). In performing the calibrations, since only one chloride concentration is used, the ideal calibration procedure couldn't be followed. Nevertheless, by assuming an initial reasonable value for $A_{0}$ and $B_{0}$, they were easily fine tuned by matching the surface concentration at 14 days (Fig. 3c). Next, the extent was matched by calibrating $\tau_{a}$ and then the curvature was matched by calibrating $f_{v}$ following the ideal calibration procedure.

The calibrated parameters values are shown in Table 2 (column 1). It should be noted that, although $\mathrm{KI}$ solution was used in the experiment the calibration is based on $\mathrm{NaCl}$ since the anions in the two solutions have similar diffusion coefficient in concrete $[88,89]$ and the experiment 
used iodide ions to study chloride diffusion because Iodine has higher fluorescence and thus, easier to measure using X-ray techniques. So, by using the calibrated parameters, simulations of the profiles at 5 and 8 days are used as validations (see Fig. 3a and Fig. 3b). By examining the surface concentrations at 5 and 8 days, it is clear that the chosen values for $A_{0}$ and $B_{0}$ seem reasonable since the surface concentrations match exactly the same surface concentration at 14 days.

Note that due to the discrete heterogeneous geometry of the diffusion network, the numerical value of the chloride concentration varies even at the same cutting section. Therefore, in all the plots in Fig. 3, the simulated scatter is reported (grey area) together with the average value of the numerical chloride concentration results that can be compared with the experimental data. This comparison shows a very good match since the numerical simulations capture pretty well the growth of the chloride concentration as the ponding time increases. It is important to notice here that the mesh used was generated using a fictitious aggregate size distribution with a maximum aggregate size of $0.9 \mathrm{~mm}$ and a minimum of $0.45 \mathrm{~mm}$ with 0.6 Fuller coefficient. This guarantees simulating $34 \%$ of the coarse aggregate as typically recommended in LDPM literature [80, 81 , $90,91]$. The maximum aggregate size was chosen to be no more than $10 \%$ of the minimum sample dimension $(9.5 \mathrm{~mm})$ in order to reduce the scatter. Nevertheless, due to the discrete nature of the model, there will always be a small scatter but to keep the computational cost reasonable, the chosen maximum aggregate size was slightly lower than $10 \%$ of the minimum dimension. Yet, our preliminary simulations show that if a coarser mesh is used, the scatter will increase but the average line stays almost the same which validates the acquired results and proves that the scatter is not an artifact but it represents a meso-scale measure of heterogeneity. The resulting conduit transport networks are shown in Fig. 4 and are colored based on the weight percentage of iodine in the cement paste.

\subsection{Diffusion in saturated cement paste: Effect of ponding temperature}

The second experimental investigation considered is described in [22]. They casted cylindrical specimens with ordinary Canadian Type 10 portland cement (diameter $=70 \mathrm{~mm}$, height $=200 \mathrm{~mm}$, cement content $\left.=380 \mathrm{~kg} / \mathrm{m}^{3}, w / c=0.6\right)$. After three months of curing in $100 \%$ 
relative humidity fog room, the samples were cut into 50 -mm disks and were sealed on the bottom and on the lateral surface and then immersed for 100 days in $0.5 \mathrm{~mol} / \mathrm{L} \mathrm{NaCl}$ solution at different temperatures, $4{ }^{\circ} \mathrm{C}, 23{ }^{\circ} \mathrm{C}$ and $38^{\circ} \mathrm{C}$. Similarly here, only the profiles relevant to a single chloride concentration are available, thus, the same procedure as specified in the previous section is used. First, the chloride penetration profile at room temperature, $23{ }^{\circ} \mathrm{C}$, is used to calibrate the 4 basic parameters, and then the experimental results at $4{ }^{\circ} \mathrm{C}$ are used to calibrate the two activation energies, $U$ and $U_{b}$. All the calibrated parameters values are reported in Table 2 (column 2). Figure 5 shows the comparisons between numerical solutions and experimental data, in which the agreement is very good. As temperature grows from $4{ }^{\circ} \mathrm{C}$ to $23{ }^{\circ} \mathrm{C}$, the chloride concentration increases and is captured by the model. Nevertheless, at $38{ }^{\circ} \mathrm{C}$, the concentration of chloride ions near the top end is slightly lower than the concentration for $23{ }^{\circ} \mathrm{C}$, contrary to what one would expect. Despite this small inconsistency in the experimental data, the numerical values of the chloride concentration present the expected trend near the top end and match pretty well the experimental data at deeper depth. It is important to note here that the provided experimental data represents an average value of chloride contents determined using ASTM C1556 [92] layer-by-layer acid dissolution procedure at 2-3 mm depth increments. Therefore, experimental scatter and resolution inaccuracy is expected, and thus, the observed slight deviation of the expected increasing trend of chloride concentration at the surface with increased temperature is very well acceptable. Similarly, here also, the conduit transport network is generated using an aggregate size range of $3 \mathrm{~mm}$ to $6 \mathrm{~mm}$ with the maximum aggregate size slightly lower than $10 \%$ of the minimum dimension $(70 \mathrm{~mm})$. Again, the gray area representing simulation scatter is also very narrow. The distribution of chloride ions within the transport network in the three concrete samples is presented in Fig. 6 at the different exposure temperatures. Note that in all figures since the chloride ions did not diffuse through the whole depth, only the active part is shown. 
(a)

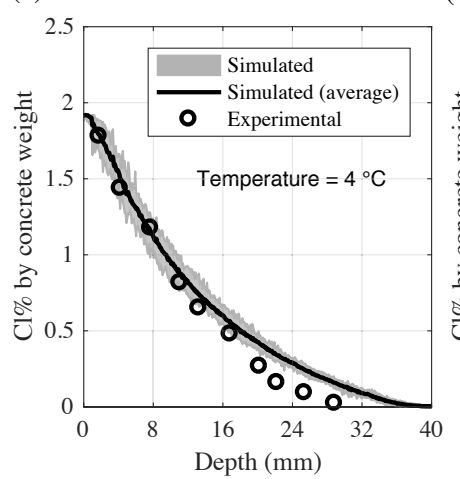

(b)

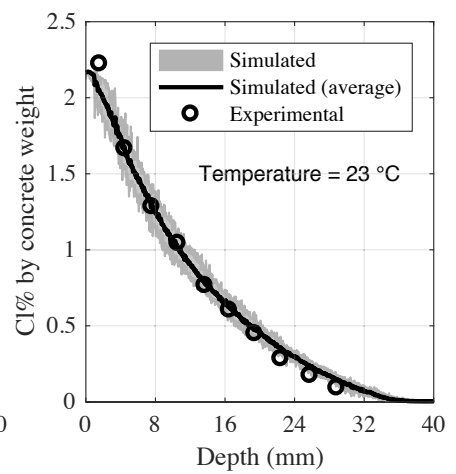

(c)

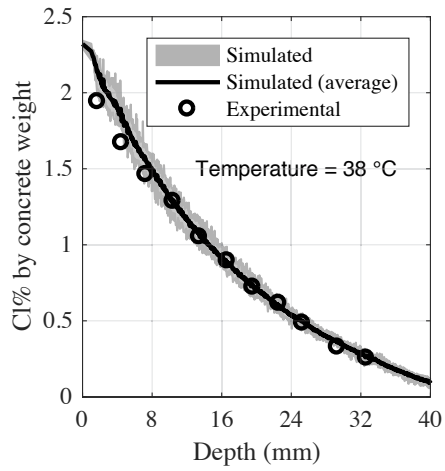

Figure 5: Experimental [22] and numerical results of chloride penetration at different temperatures: a) $4{ }^{\circ} \mathrm{C}$; b) $23{ }^{\circ} \mathrm{C}$; c) $38^{\circ} \mathrm{C}$

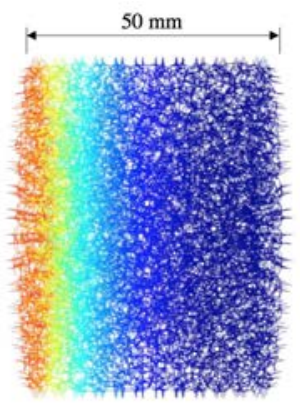

$4{ }^{\circ} \mathrm{C}$

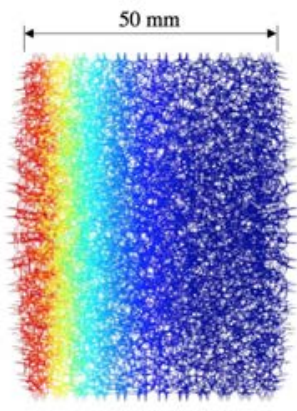

$23^{\circ} \mathrm{C}$

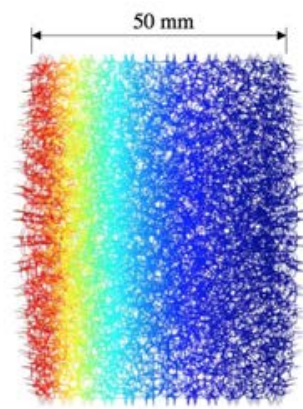

$38^{\circ} \mathrm{C}$

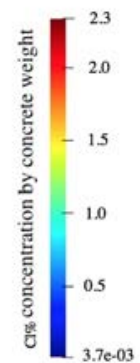

Figure 6: Contour of chloride concentration at different temperatures from simulation

\subsection{Diffusion in saturated concrete}

Ordinary concrete is a multi-phase composite material in which the aggregate pieces are relatively impermeable and any diffusion process takes place mostly in the cement paste and interfacial transition zone. Many researchers [16, 20,93] investigated the influence of aggregate content on chloride ingress and concluded that the addition of aggregate does influence chloride diffusion due to the different overall porosity of concrete as compared to that of cement paste. In the proposed model the porosity of concrete $\phi$ is analyzed based on the HTC model and the influence of aggregate on the diffusion path of chloride is considered by tortuosity parameter $\tau_{a}$ in Eq. 23. 
(a)

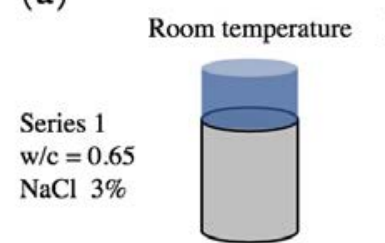

C1

Series 2 $\mathrm{w} / \mathrm{c}=0.65$ $\mathrm{NaCl} 5 \%$

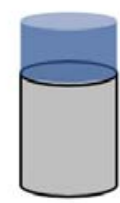

C4

Series 3

$\mathrm{w} / \mathrm{c}=0.55$

$\mathrm{NaCl}=3 \%$

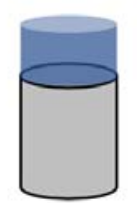

C7

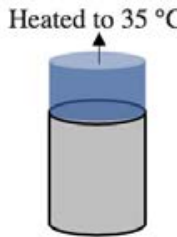

C2

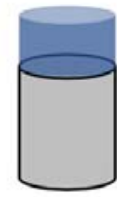

C5

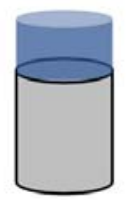

C8

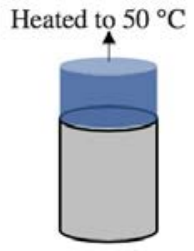

C3

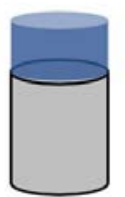

C6

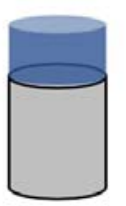

C9 (b)

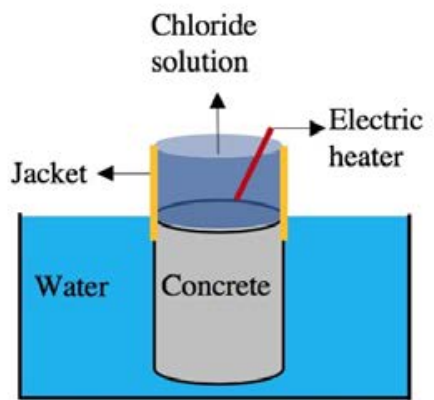

Figure 7: Experimental investigation from [23] (a) Testing series and experimental parameters (b) Experimental setup

In the previous sections, the effect of aggregate is not considered since the numerical analyses concern only cement paste. Herein the effectiveness of the model in simulating concrete is analyzed with reference to a complete experimental study conducted by Isteita and Xi [23]. They performed ponding tests, summarized in Figure 7a, that comprised three series exposed to different temperature gradients and chloride concentrations. There were three specimens of the same mix in all three series and all of them were cylinders with $150 \mathrm{~mm}$ in diameter and 200 $\mathrm{mm}$ in height. The first and second series used a mix that contains $356 \mathrm{~kg} / \mathrm{m}^{3}$ of cement (ASTM C150 Type I) with $0.65 w / c$ and 5.28 aggregate to cement ratio. The third series used a mix that contains $380 \mathrm{~kg} / \mathrm{m}^{3}$ of cement (ASTM C150 Type I) with $0.55 \mathrm{w} / \mathrm{c}$ and 4.24 aggregate to cement ratio. For both mixes, aggregate size distribution data was not reported so, a maximum aggregate size of $19 \mathrm{~mm}$, a minimum aggregate size of $5 \mathrm{~mm}$, and a fuller coefficient of 0.5 are assumed in the simulation for such a typical concrete mixture. Using these data one can construct the LDPM mesh and then create the conduit mesh as explained before. Note here that all coarse aggregate volume was represented since the chosen minimum aggregate size is the typical limit between 
coarse and fine aggregate. This guarantees that the generated conduit network is rich enough so that the conduits are passing throughout mortar only.

After twenty-eight-day curing all samples were immersed in distilled water (see Fig.7b) and ponding tests were performed from the top ends with a solution of $3 \%, 5 \%$ and $3 \%$ chloride concentration (mass fraction) for the three series, respectively, at three constant temperatures of $23{ }^{\circ} \mathrm{C}, 35^{\circ} \mathrm{C}$ and $50{ }^{\circ} \mathrm{C}$ in each series. The chloride concentration at different depths was evaluated for five different durations of ponding: 3 days, 6 days, 12 days, 24 days, and 48 days Since the temperature of the distilled water is relatively constant, $20^{\circ} \mathrm{C}$, the ponding tests were thus performed under a temperature gradient. More details on the experimental investigation can be found in [23].

In the numerical simulation to accurately impose the temperature boundary conditions, the temperature of the top surface of the specimen is set as the corresponding temperature of the chloride solution whereas the temperature of the other external surfaces is set at the initial environmental temperature of $20^{\circ} \mathrm{C}$ and then kept constant. Different from previous experimental investigations which are strictly 1-D transport, the setup in this experiment might cause a non-uniform transport of chloride ions along a cross-section due to the temperature difference between the side surface and the inside core. As the penetration goes on, a temperature gradient along the cross section of the cylinder is created. Chloride ions near the core concrete transport faster due to a higher temperature, compared with those near the side surface. As the ponding test continues, the differences accumulate gradually, resulting in a non-uniform chloride distribution along the cross section. To visualize this profile, the chloride concentration field was interpolated from the diffusion network on a tetrahedral mesh sharing the same diffusion network nodes and then the volume was colored by the field values as shown in Fig. 8. One can see the slice taken at $13 \mathrm{~mm}$ depth showing clearly the higher chloride concentrations in the middle. Note also the ability of the model to scatter the concentrations across the specimen. As opposed to any other continuum model, while there is an obvious concentration gradient from the center towards the edge (See Fig. 8a), the gradient is not axisymmetric but rather shows irregular variations. These variations are due to the scatter of the diffusion network around the aggregate. It is also impor- 
tant to mention that in the experiments, concrete powder was collected by drilling a small hole at the center of the sample then, this powder was used to determine the chloride concentration using ASTM C1152/C1152M-04(2012) [92]. To mimic the experiments numerically, only the numerical results within an inner core with one third of the original radius are considered when analyzing the chloride concentration profile. The one third value was chosen by best guess since the drilling diameter was not reported in the experiments.

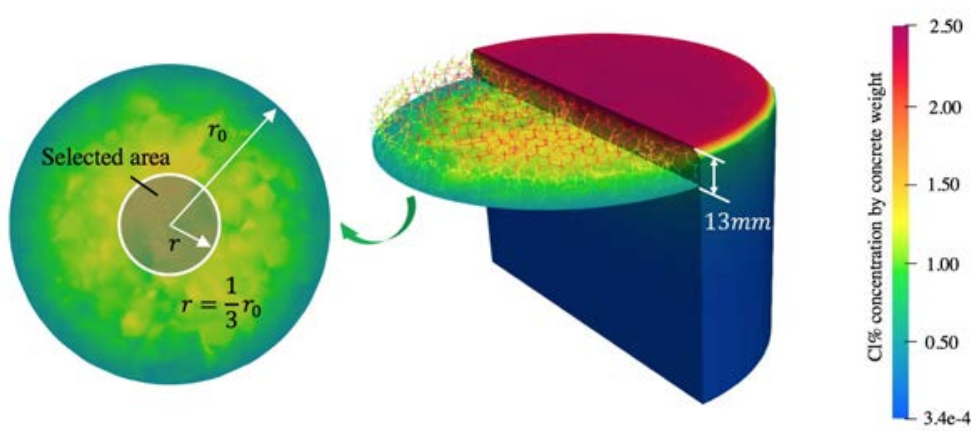

Figure 8: Numerical results of chloride distribution of sample C3 after 48-day ponding. cross section at depth $=13 \mathrm{~mm}$, vertical section along central axis.

To perform a more accurate calibration, the four basic parameters $\tau_{a}, A_{0}, B_{0}$ and $f_{v}$ were calibrated by fitting the 3-day and the 48-day ponding curves obtained under room temperature, $23{ }^{\circ} \mathrm{C}$, in series 1 and 2 (with $3 \%$ and $5 \%$ chloride concentrations, respectively). The corresponding best fitting plots are shown in Figs. 9a and 9e and Figs. 10a and 10e. Then activation energy $U$ and $U_{b}$ are calibrated by fitting the 48-day experimental data under temperature of $35{ }^{\circ} \mathrm{C}$ in series 1 , as shown in Fig. 9e. In this calibration, five curves were used instead of the ideal three curves mentioned in the calibration procedure. This was because of the large scatter of the experimental data. 
(a)

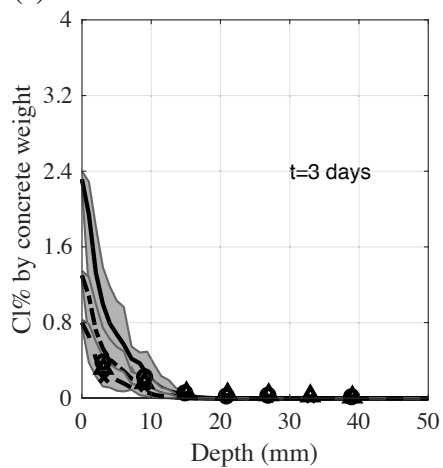

(d)

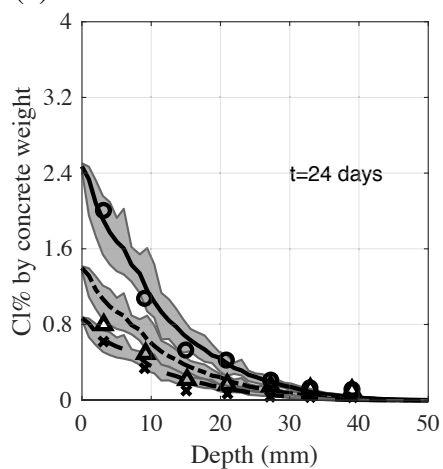

(b)

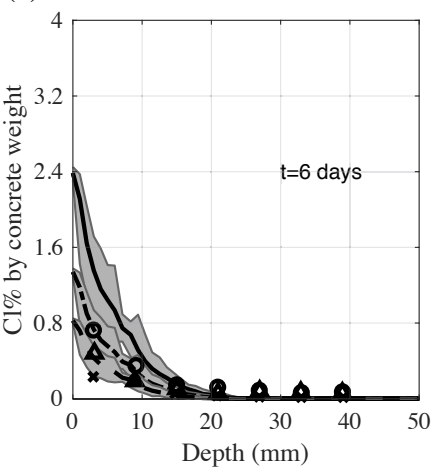

(e)

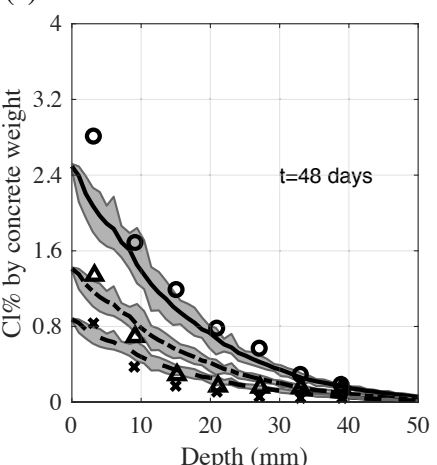

(c)

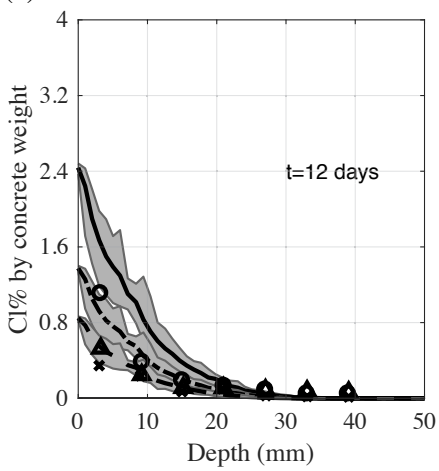

Depth (mm)

$$
\begin{aligned}
& \text { Simulated } \\
\hline & \text { Simulated (average, } \left.50^{\circ} \mathrm{C}, \mathrm{C} 3\right) \\
\hline-- & \text { Simulated (average, } \left.35^{\circ} \mathrm{C}, \mathrm{C} 2\right) \\
\mathbf{-} & \text { Simulated (average, room temp., C1) } \\
\mathbf{0} & \text { Experimental }\left(50^{\circ} \mathrm{C}, \mathrm{C} 3\right) \\
\mathbf{\Delta} & \text { Experimental }\left(35^{\circ} \mathrm{C}, \mathrm{C} 2\right) \\
\mathbf{\times} & \text { Experimental (room temp., C1) }
\end{aligned}
$$

Figure 9: $\mathrm{C} 1 \sim \mathrm{C} 3$ experimental [23] and numerically simulated chloride penetration for different ponding durations, $w / c=0.65, \mathrm{NaCl}$ concentration $=3 \%$ : (a) 3 days; (b) 6 days; (c) 12 days; (d) 24 days; (e) 48 days. 
(a)

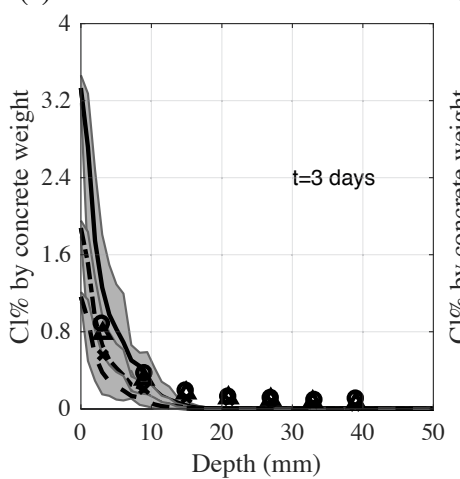

(d)

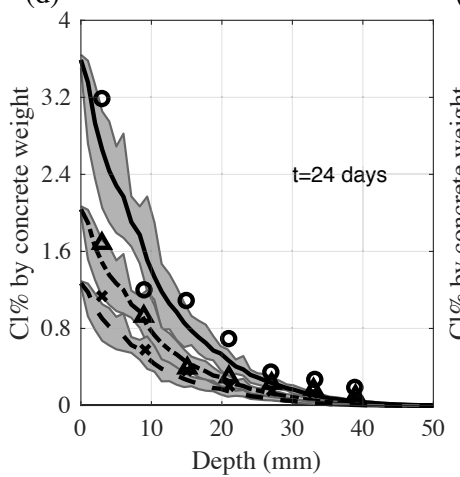

(b)

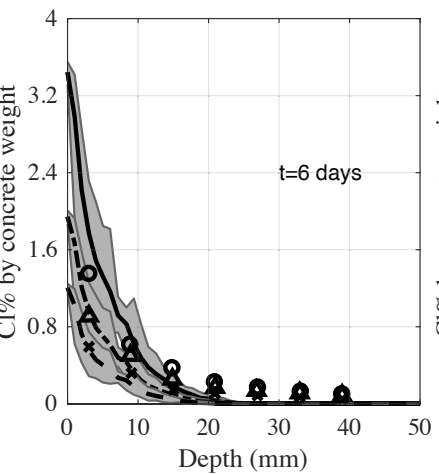

(e)

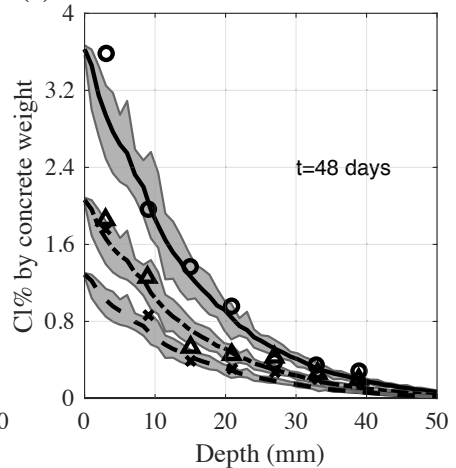

(c)

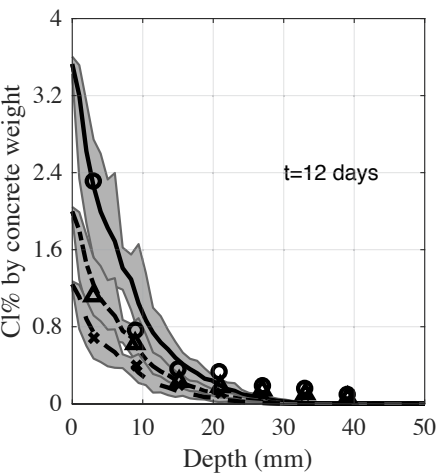

Figure 10: C4 C6 experimental [23] and numerically simulated chloride penetration for different ponding durations, $w / c=0.65, \mathrm{NaCl}$ concentration $=3 \%$ : (a) 3 days; (b) 6 days; (c) 12 days; (d) 24 days; (e) 48 days. 
(a)

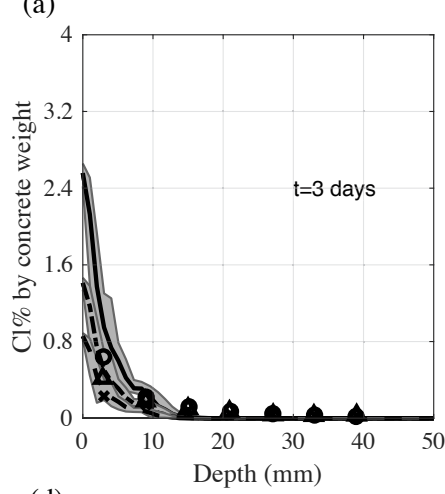

(d)

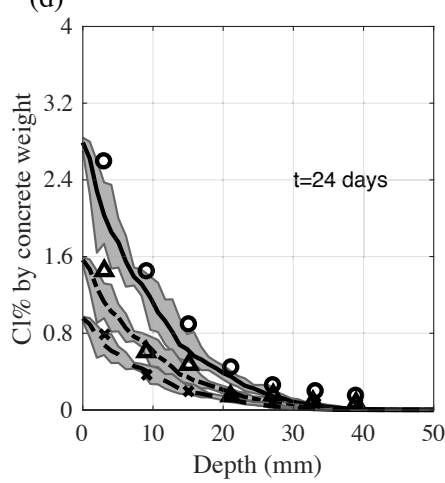

(b)

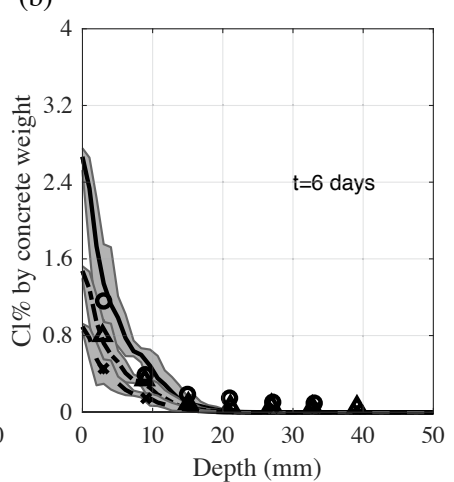

(e)

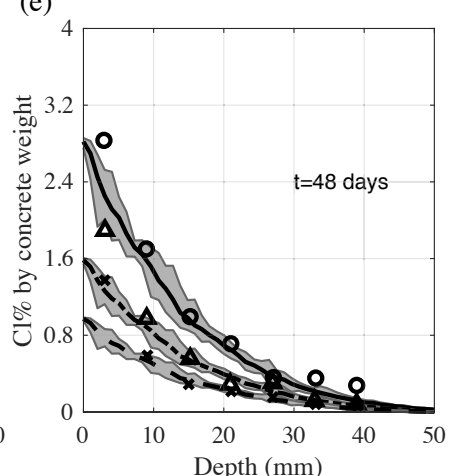

(c)

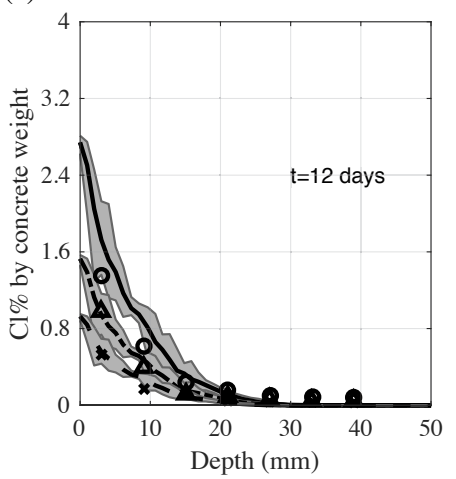

Simulated

Simulated (average, $50^{\circ} \mathrm{C}, \mathrm{C} 9$ ) -- - S Simulated (average, $35^{\circ} \mathrm{C}, \mathrm{C} 8$ )

- Simulated (average, room temp., C7)

- Experimental $\left(50^{\circ} \mathrm{C}, \mathrm{C} 9\right)$

$\Delta$ Experimental $\left(35^{\circ} \mathrm{C}, \mathrm{C} 8\right)$

* Experimental (room temp., C7)

Figure 11: C7 C9 experimental [23] and numerically simulated chloride penetration for different ponding durations, $w / c=0.65, \mathrm{NaCl}$ concentration $=3 \%$ : (a) 3 days; (b) 6 days; (c) 12 days; (d) 24 days; (e) 48 days.

It should be noted that although series 3 has different $w / c$ from series 1 and 2 , it is assumed that the pore structure was shaped with similar tortuosity since the $w / c$ are close $(0.55$ and 0.65$)$.

Thus, the effect of $w / c$ was totally lumped in the porosity (acquired from the HTC model) and the same apparent tortuosity factor is employed for series 3. Additionally, the values of the material parameters $A_{0}$ and $B_{0}$ are are kept unchanged according to the findings in [45], which shows that the bound chloride in relation to the free chloride could be considered independent from the water-cement ratio if the amount of bound chloride is re-scaled per cement content. By using the same parameter values in all the series, it is shown that the proposed model is capable of capturing automatically the influence of different $w / c$. Nevertheless, if the $w / c$ varies significantly, it is expected that the pore structure characteristics may be more noticeably different and thus, the apparent tortuosity factor may need to be recalibrated. All calibrated values are listed in Table 2 
(column 3). As a strong validation of the model, all simulations of the remaining experimental results serve as validations.

The validation of the model is presented in Fig. 9 11 by comparing experimental and numerical results. The agreement is overall excellent, which proves that the proposed model is able to predict the chloride ingress in concrete. Fig. $9 \sim 11$ show, for various temperature gradients, the chloride penetration after ponding for $3,6,12,24$, and 48 days. As it can be observed in the plots, the higher the temperature gradient is, the faster the chloride ions ingress and the more chloride ions get bound into the gel. This proves that the temperature effect has to be considered using two different activation energies as proposed by the model for the diffusion and binding through the exponential coefficient presented in Eq. 5 and Eq. 25.

Fig.10 shows the chloride profile in series 2 test, which had higher concentration of $\mathrm{NaCl}$ on the boundary (5\%) than series 1 and the same other boundary conditions. So, as expected, the three samples of series 2 present an higher chloride concentration profile than the corresponding samples in series 1 for each ponding duration. This feature is well captured by the numerical simulation using the same parameters as in series 1 .

Finally, Fig. 11 shows the chloride penetration profile of series 3 for the samples $\mathrm{C} 7 \sim \mathrm{C} 9$ with a lower water-cement ratio of 0.55 and the same boundary conditions as in series 1 . Also in this case the numerical analyses are performed using the same parameters as discussed before without any changes. Comparing the experimentally measured chloride profiles in series 1 (Fig. 9) and series 3 (Fig. 11), the chloride concentration near the top surface of samples are relatively higher in series 3 than that in series 1 . This can be caused by the increase of hydrated cement content in series 3, which increases the amount of chloride ions bound to the matrix, although the lower $w / c$ produces a relatively smaller porosity that reduces the chloride diffusivity. This difference reaches its maximum at the top surface that is immersed in the external chloride solution with the free chloride content almost constant. This behavior is fully captured by the proposed the model as shown by Fig. 12 in which the free (Fig. 12a) and bound (Fig. 12b) chloride contents in the two series are compared. As shown in the Fig. 12, close to the exposed surface, both free and bound chloride concentrations in sample C8 are higher than that in sample 
C2. Considering the fact that the concentration of bound chloride is higher than free chloride by about an order of magnitude, the increased total chloride concentration in sample C8 is mainly attributed to the binding process. Farther away from the exposed external surface, bound chloride and free chloride concentration in sample $\mathrm{C} 2$ and $\mathrm{C} 8$ become nearly the same, implying that the chloride penetration front is not substantially affected by the change of pore structure due to $w / c$ difference. This observation also supports the use of a constant tortuosity factor for all series samples.

(a)

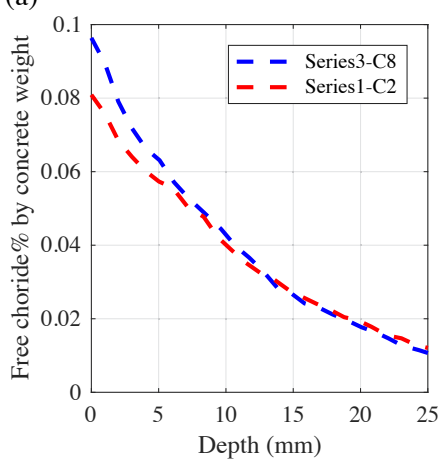

(b)

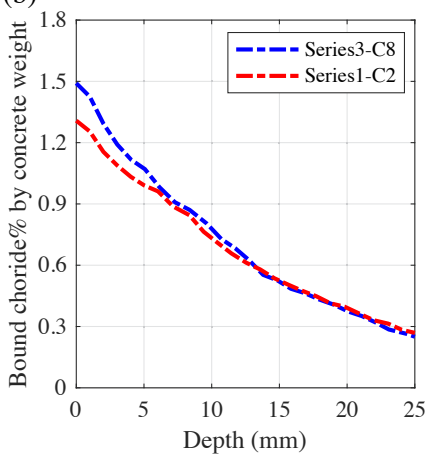

Figure 12: Comparison of free and bound chloride: (a) Free chloride in sample C2 and C8. (b) Bound chloride in sample $\mathrm{C} 2$ and $\mathrm{C} 8$

To summarize the observations from the simulation of this large set of experiments, the envelops between 6 and 48 days of ponding of both experimental and numerically simulated chloride concentration profiles for the three series at all three temperatures are shown in Fig. 13. Note that only the data from C1 (Fig. 13d), C4 (Fig. 13g) and C2 (Fig. 13e) are used for model calibration. As it can be seen, the maximum range of concentrations in these data doesn't exceed $1.5 \% \mathrm{Cl}$ concentration by concrete weight, however, the model is able to predict values as high as $3.6 \% \mathrm{Cl}$ concentration by concrete weight for $\mathrm{C} 6$ (Fig. 13i). It is clear that the model can capture the effects of increasing temperature (going from left to right in Fig. 13) and increase in $w / c$ as well as chloride boundary concentrations (going from top to bottom in Fig. 13). It is also interesting to notice that the experimental value closest to the top surface of the sample at 48 days seems to be higher than the predicted numerical one. As discussed before, that can be due to the increased errors in evaluating the concentration at the surface since it is in direct contact with 
768 the solution, but also, another reason can be due to the small cracks that may have developed on 769 the sample surface during early age before demolding and full saturation. Nevertheless, the rest 770 of the envelop points are in very good agreement with the numerical simulations which shows 771 that the model is not a mere extrapolation but actually captures mechanisms beyond the ranges 772 of data it was calibrated with. 
(a)

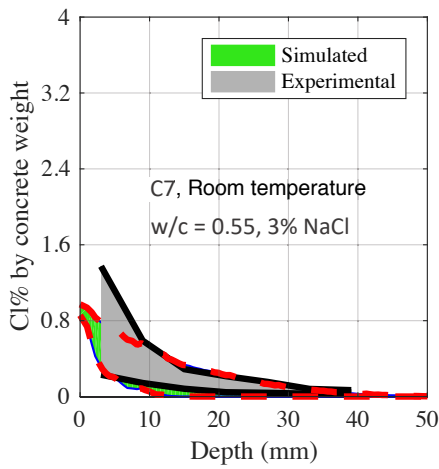

(d)

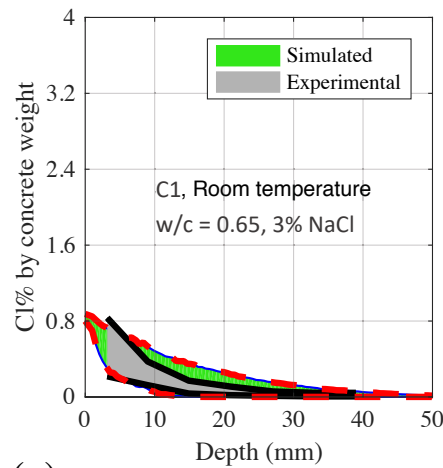

(g)

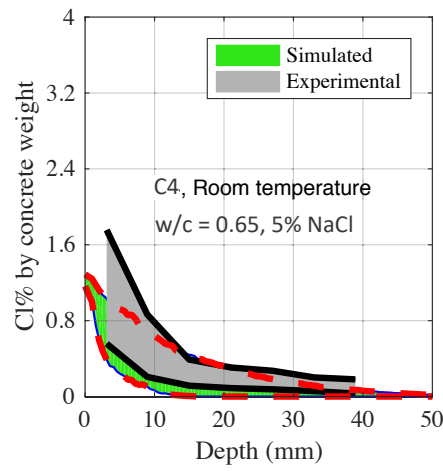

(b)

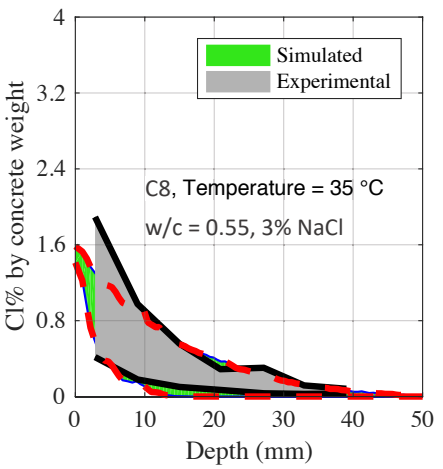

(e)

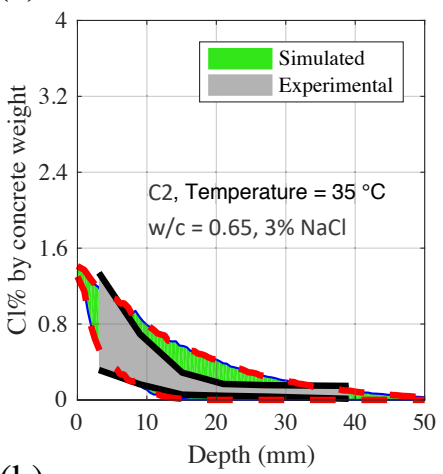

(h)

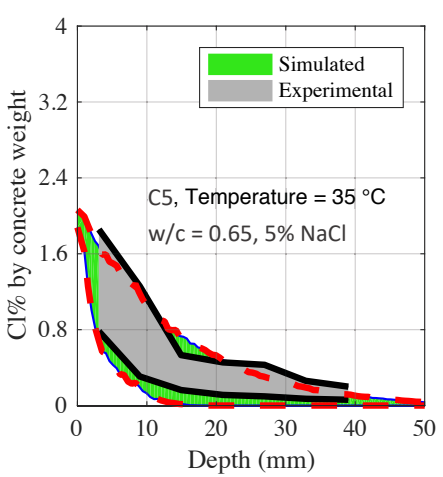

(c)

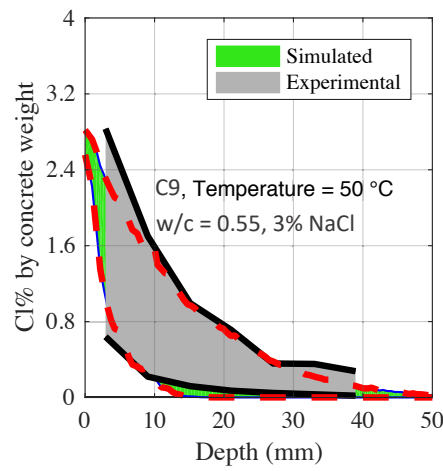

(f)

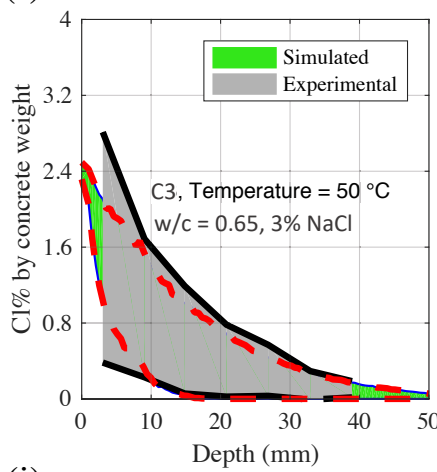

(i)

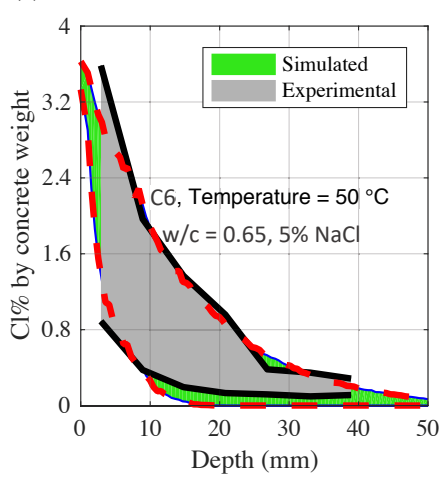

Figure 13: Envelop of experimental [23] and numerically simulated chloride concentration profiles for Series 3 a) to c), Series $1 \mathrm{~d}$ ) to f), and Series $2 \mathrm{~g}$ ) to i)

\subsection{Diffusion in unsaturated concrete}

For unsaturated concrete, the advection effect contributes to the transport of chloride ions, leading to different chloride concentration profiles comparing with the saturated condition, as 
clearly shown in [24]. To verify that the proposed formulation is also capable of characterizing the transport of chloride ions in unsaturated conditions, the numerical simulations of the experiments in [24] are carried out and the results are compared with the experimental output. As summarized in Table 3, two groups of specimens made of the same mixture were adopted in the study. The mix contained $380 \mathrm{~kg} / \mathrm{m}^{3}$ of cement (ASTM C150 Type I) with $0.55 \mathrm{w} / \mathrm{c}$ and 4.24 aggregate to cement ratio. Maximum and minimum aggregate size are assumed to be $19 \mathrm{~mm}$ and $5 \mathrm{~mm}$, respectively and a fuller curve coefficient of 0.5 is used, similarly to the previous section.

After 28 days of curing, the first group of samples was immersed in water for 30 days to reach full saturation, while the second group of samples was exposed to air in laboratory environment, with a temperature of $22.8{ }^{\circ} \mathrm{C}$ and relative humidity of $32 \%$. Then both groups were exposed to $3 \%$ and $5 \% \mathrm{NaCl}$ solution at the top surface and distilled water at the bottom surface. Relative humidity of samples in group 2 was monitored at depth of 1 in $(25.4 \mathrm{~mm})$ and 2 in $(50.8 \mathrm{~mm})$.

To calibrate the model, first, the HTC model parameters relevant to moisture diffusion have to be calibrated since in this experimental campaign, moisture will move under the humidity gradients. These parameters are $D_{h 0}, D_{h 1}$ and $n$. For all other campaigns these parameters are not effective since no moisture diffusion is occurring at full saturation. These parameters were calibrated by using the humidity profiles from group 2. Note that for full calibration of the other HTC parameters, more experimental data would have been needed and thus, the calibration started by assuming all other HTC model parameters to be the same as those listed in Table 1 . Calibration results give $D_{h 0}=8.05 \times 10^{-8} \mathrm{~m}^{2} / \mathrm{h}, D_{h 1}=2.37 \times 10^{-6} \mathrm{~m}^{2} / \mathrm{h}$ and $n=5.0$. As it can be seen from Fig. 14, the HTC model can capture very well the changes in the moisture content of the unsaturated concrete and its evolution over time.

Table 3: Testing series and experimental data of [23]

\begin{tabular}{|l|l|lc|}
\hline Group & \multicolumn{2}{|c|}{1} & \multicolumn{2}{|c|}{2} \\
\hline Sample number & SC1 SC2 & UC1 UC2 \\
\hline Saturation condition & saturated & unsaturated \\
\hline Cl concentration at the boundary & $3 \% \quad 5 \%$ & $3 \% \quad 5 \%$ \\
\hline
\end{tabular}


(a)

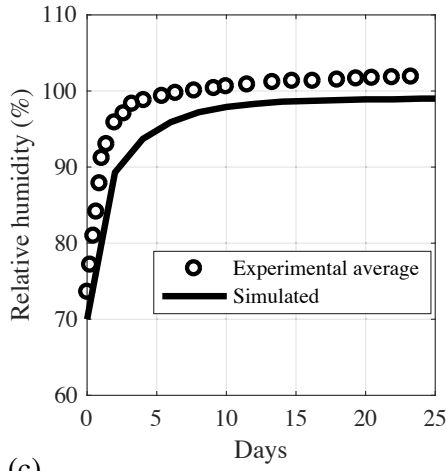

(c)

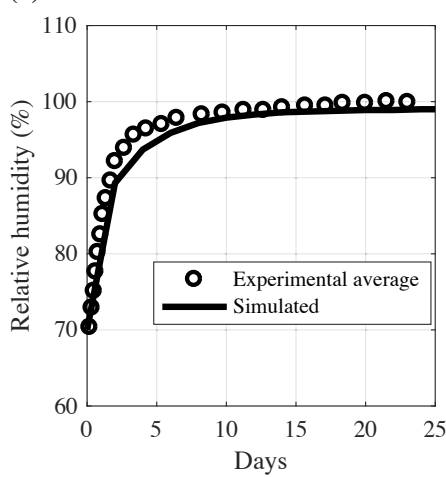

(b)

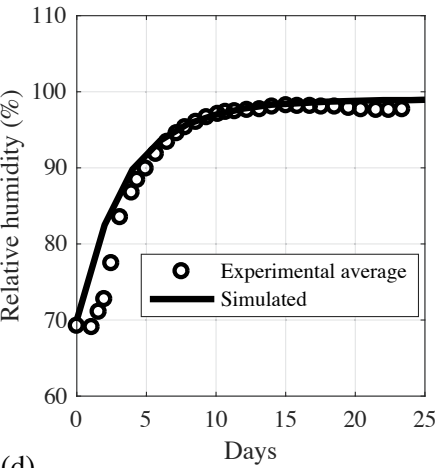

(d)

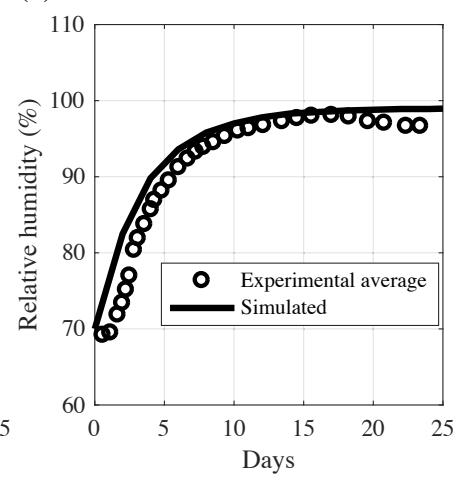

Figure 14: Experimental [24] and numerically simulated relative humidity evolution. (a) Depth $=1$ in., $\mathrm{NaCl}$ concentration $=3 \%$, (b) Depth $=2$ in., $\mathrm{NaCl}$ concentration $=3 \%$, (c) Depth $=1$ in., $\mathrm{NaCl}$ concentration $=5 \%$, (d) Depth $=2$ in., $\mathrm{NaCl}$ concentration $=5 \%$

As shown in Fig. 14a, the experimental relative humidity being obviously higher than the simulated one might be caused by measurements errors because after 15 days the recorded experimental relative humidity is above $100 \%$. In addition, typically, relative humidity sensors have large errors close to saturation that can reach up to 5\% errors. Another reason for this difference is the fact that the concrete was put in a very dry condition (only $32 \%$ relative humidity) to speed up the moisture removal, as a result, some surface drying cracks must have formed and that would have increased the relative humidity close to the surface which explains why the model predictions at 1 in depth are slightly below the experimental values (see Fig. 14a,c).

Once the HTC model parameters are calibrated, the chloride penetration profile of the saturated specimen exposed to $3 \%$ and $5 \% \mathrm{NaCl}$ solution for 30 days (see Fig. 15a,c) is then adopted to calibrate the chloride diffusion parameters following the ideal calibration procedure. Since 
no temperature gradients exist in this set, the activation energies can not be calibrated and their effects are not considered (since $T=T_{0}$ ). Calibrated parameters are displayed in Table 2 (column 4). Similarly here, all the remaining experimental data serve as validation including the two other penetration curves at full saturation and the four penetration curves at partial saturation.

The effects of the humidity gradients are clearly captured by the model. When the concrete is unsaturated, the gradient of relative humidity causes advection and contributes to the flux speed, leading to a faster transport of chloride than that in saturated concrete, which is a fact that can be easily observed by comparing Fig. 15 and Fig. 16. Note also here that the model captures the scatter again within the concrete sample which is an important feature that is lacking in macroscopic continuum models.

As in the previously considered cases, the proposed model captures very well the chloride transport under different chloride concentrations and for different ponding durations (Fig. 15). More importantly, for unsaturated concrete, with the same parameter values, the proposed model is capable of predicting quantitatively the chloride transport under different chloride concentrations and for different ponding durations too (see Fig. 16). This emphasizes the fact that the model is correctly representing the physical transport processes within the pore solution and therefore, it predicts the concentration correctly by just adding the convective part without the need to adjust the chloride diffusion coefficient $D_{c}$. In other words, $D_{c}$ as predicted by the model accounts correctly for all physical phenomena that occur within the concentrated concrete pore solution as it moves through the porous structure and links them correctly to a single constant chloride diffusion coefficient in dilute solution $D_{0}$. 
(a)

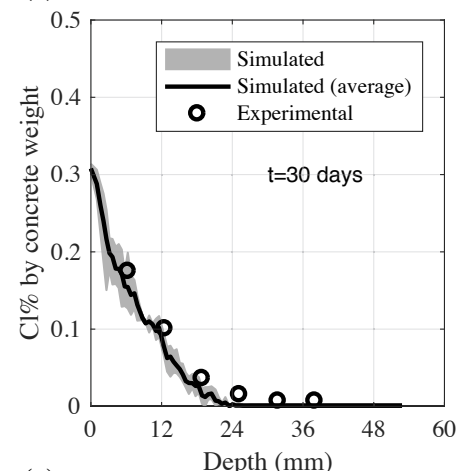

(c)

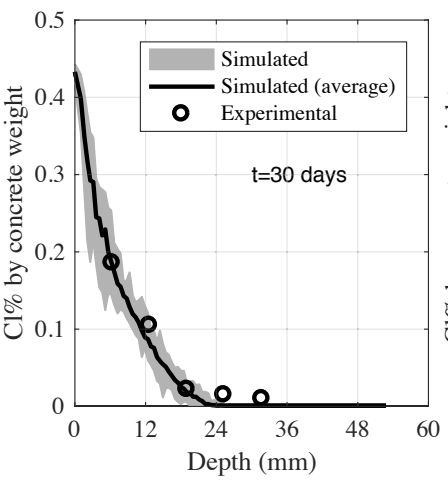

(b)

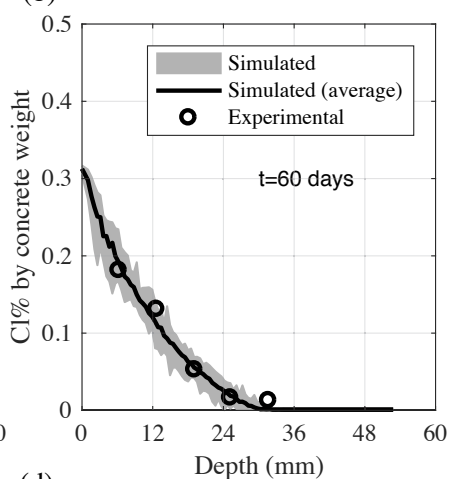

(d)

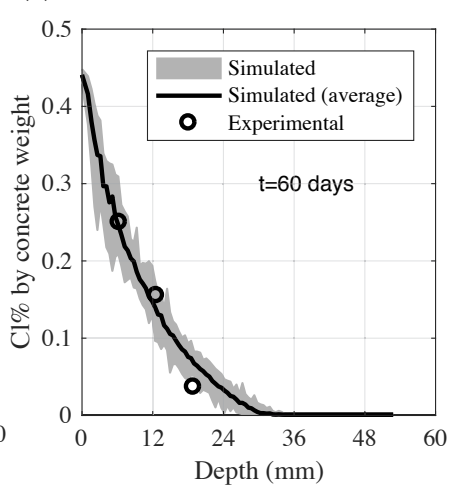

Figure 15: Experimental [24] and numerically simulated chloride penetration for fully saturated specimens at different ponding days. (a) and (b): Specimens exposed to $3 \% \mathrm{NaCl}$ solution (SC1), (c) and (d): Specimens exposed $5 \%$ to $\mathrm{NaCl}$ solution (SC2) 
(a)

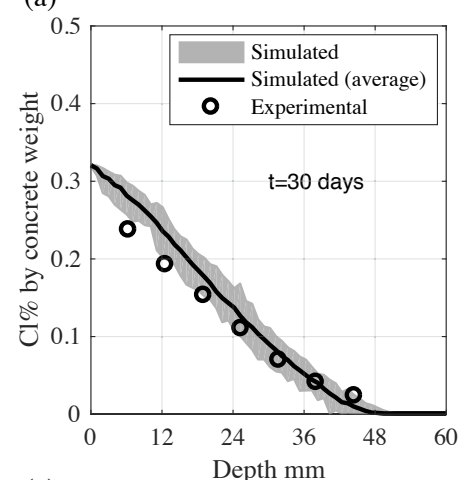

(c)

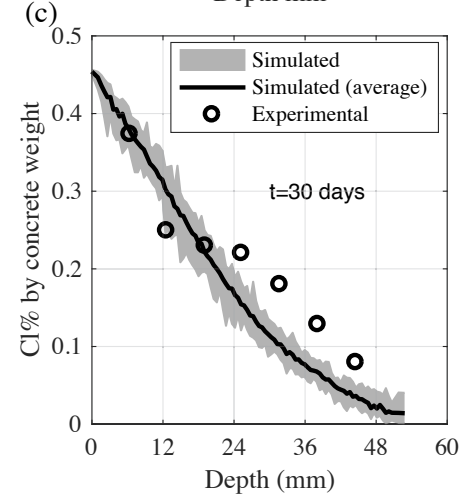

(b)

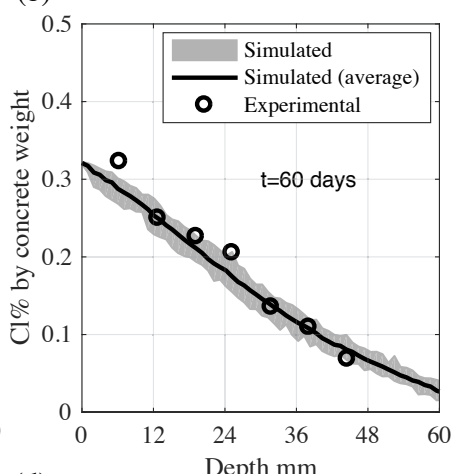

(d)

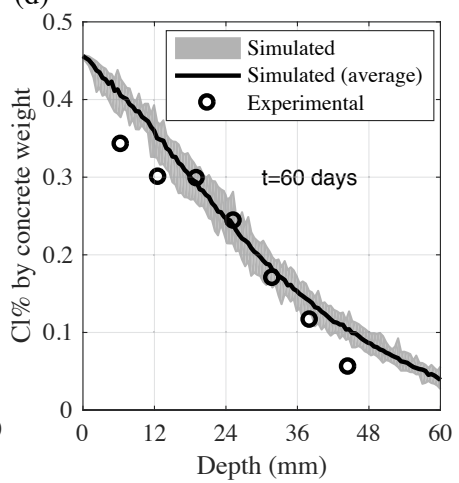

Figure 16: Experimental [24] and numerically simulated chloride penetration for unsaturated specimens at different ponding days. (a) and (b): Specimens exposed to $3 \% \mathrm{NaCl}$ solution (UC1), (c) and (d): Specimens exposed to $5 \% \mathrm{NaCl}$ solution (UC2)

\subsection{Discussion of calibration and simulation results}

Here, the postulated benefits (see Sec.2) of the modeling approach are discussed based on the numerical analyses of the considered experimental results and their corresponding calibrated parameters.

\subsubsection{Representation of inherent physics with limited model parameters}

As shown in the simulation results, the presented model can simulate accurately a wide variety of experimental data relevant to two cement pastes and two concrete mixes with a maximum aggregate size of $19 \mathrm{~mm}$. The $w / c$ varied from 0.4 to 0.6 . Chloride concentrations varied between 3\% and 5\% at the boundaries. Variable ponding durations, thermal gradients, and hygral gradients were also considered. This means that a wide variety of microstructure characteristics 
and exposure conditions are covered (not mentioning the variation in curing conditions too). For all these varieties, only four basic parameters are calibrated for isothermal conditions and only two additional parameters are calibrated to capture thermal effects. In addition, no extra parameters are used to capture the unsaturated conditions and just the correct inclusion of coupling between the thermal and hygral processes with the chloride diffusion process accounts for the unsaturated conditions differences. All other physics are considered in the modeling framework by using comprehensive formulations from literature with predefined fixed material parameters. This shows that the model successfully strikes a good balance between physics and simplicity.

\subsubsection{Robustness of the calibrated model parameters}

Table 4: Sensitivity analysis based on the simulation in Sec. 7.2

\begin{tabular}{|l|l|l|l|l|}
\hline \multicolumn{1}{|c|}{ Parameter } & $\begin{array}{l}\text { Concentration } \\
\text { at } 15 \mathrm{~mm}\end{array}$ & $\begin{array}{l}\text { Variation } \\
(\Delta C / C)\end{array}$ & $\begin{array}{l}\text { Depth of Pene- } \\
\text { tration }(\mathrm{mm})\end{array}$ & $\begin{array}{l}\text { Variation } \\
\left(\Delta d_{p} / d_{p}\right)\end{array}$ \\
\hline \multicolumn{5}{|c|}{ Temperature $=23^{\circ} \mathrm{C}$} \\
\hline$\tau_{a}(+10 \%)$ & $0.75 \%$ & $5.63 \%$ & 36.34 & $3.80 \%$ \\
$\tau_{a}(-10 \%)$ & $0.66 \%$ & $-7.04 \%$ & 32.83 & $-6.22 \%$ \\
\hline$A_{0}(+10 \%)$ & $0.67 \%$ & $-5.63 \%$ & 37.80 & $7.97 \%$ \\
$A_{0}(-10 \%)$ & $0.76 \%$ & $7.04 \%$ & 32.11 & $-8.28 \%$ \\
\hline$B_{0}(+10 \%)$ & $0.78 \%$ & $9.86 \%$ & 30.37 & $-13.25 \%$ \\
$B_{0}(-10 \%)$ & $0.63 \%$ & $-11.27 \%$ & 40.32 & $15.17 \%$ \\
\hline$f_{v}(+10 \%)$ & $0.67 \%$ & $-5.63 \%$ & 34.56 & $-1.29 \%$ \\
$f_{v}(-10 \%)$ & $0.66 \%$ & $-7.04 \%$ & 35.36 & $1.00 \%$ \\
\hline & & Temperature $=38{ }^{\circ} \mathrm{C}$ & $3.13 \%$ \\
\hline$U(+10 \%)$ & $0.99 \%$ & $0.02 \%$ & 45.89 & $-1.46 \%$ \\
\hline$U(-10 \%)$ & $0.95 \%$ & $-0.02 \%$ & 43.86 & 44.57 \\
\hline$U_{b}(+10 \%)$ & $0.97 \%$ & $0.00 \%$ & 45.02 & $0.13 \%$ \\
$U_{b}(-10 \%)$ & $0.96 \%$ & $-0.01 \%$ &
\end{tabular}


As shown in Table 2, three of the four basic parameters of the chloride diffusion model have values that vary within the same order of magnitude: $A_{0}$ is between 0.45 and $0.7, B_{0}$ is between 1.0 and 1.9 , and $f_{v}$ varies from 400 to 600 . Only the apparent tortuosity $\tau_{a}$ shows a larger range (one order of magnitude difference). By studying $\tau_{a}$ in light of the experimental data, one could infer some explanations. First, comparing the two cement pastes, the differences are not that large and are very acceptable since they were made using two different cements, a US ASTM C150 Type I cement (Sec. 7.1) compared to a Canadian CSA T10 cement (Sec. 7.2). So, the slight difference between the two general purpose cements may be the reason for such range. Additionally, controlling bleeding is very challenging when making cement paste especially when $w / c$ is high. Therefore, the increased diffusivity identified by a higher $\tau_{a}$ in the second set (Sec. 7.2) could be a result of some bleeding that was inevitable in making the paste. Note that this does not affect the concrete and this is why, the same $\tau_{a}$ represented well all samples in the third experimental campaign (Sec. 7.3). As for the two concrete experiments, the difference in $\tau_{a}$ is relatively large although they have similar $w / c(0.55$ and 0.65$)$. Here one must consider the contribution of multiple factors. By comparing the two sets and especially Series 3 in Sec. 7.3 with SC1 in Sec. 7.4, both have identical mix designs and are exposed to the same chloride concentrations yet, the surface chloride \% content is significantly different: C7 (from Series 3 at room temperature) has about 1\% content (see Fig. 11), while SC1 has a $\mathrm{Cl} \%$ of 0.32 (see Fig. 15a and b) which is almost one third that of $\mathrm{C} 7$. Therefore it is expected that the preparation and/or curing of the two experimental campaigns is the reason for such an observed difference in their experimental results. This observed difference explains why $A_{0}$ and $B_{0}$ are higher for Sec. 7.3 (to account for the higher surface concentration) and also provides some explanation to why $\tau_{a}$ is also higher. In addition, by comparing the extent of the chloride concentration-depth profiles for C7 at 48 days (see Fig. 11e in Sec. 7.3) and SC1 at 60 days (see Fig. $15 \mathrm{~b}$ in Sec. 7.4), it can be seen that the penetration went beyond $40 \mathrm{~mm}$ after only 48 days for $\mathrm{C} 7$ and was less than $36 \mathrm{~mm}$ for SC1 after 60 days. Thus the depth of penetration is clearly larger in C7 and this requires a larger $\tau_{a}$ as previously explained. So, this proves that the model parameters are robust since within one experimental campaign Sec. 7.3) a single set of model 
parameters is used but it does cover a wide range of penetration profiles under varying $w / c$, chloride concentrations at the boundaries and temperature conditions even with a nonuniform diffusion profile.

In regards to the thermal effect parameters $U$ and $U_{b}$, the fact that only one additional curve is used to calibrate them in the third experimental set (Sec. 7.3) and the results are valid for 40 other curves prove the robustness of these two parameters too. Note that 9 test cases $\mathrm{C} 1$ to $\mathrm{C} 9$ with 5 different ponding durations are simulated (45 curves) after calibration by using only 5 curves with 4 curves to calibrate the 4 basic parameters and fine tune them, then only 1 curve to calibrate $U$ and $U_{b}$, and then (45 - $5=$ =) 40 curves for validation.

To further study the robustness and sensitivity of the calibrated parameters, a sensitivity analysis of the parameter values is performed based on the numerical simulation of the second experimental investigation (Sec. 7.2). The chloride penetration model parameters in Table 2 (column 2) are separately varied by $\pm 10 \%$. Simulations of the effects of varying the 4 basic parameters are performed using the case at room temperature. The activation energy parameters have to be investigated at a temperature other than $T_{0}$, which is assumed to be the room temperature and thus, they are tested using the simulation at $38{ }^{\circ} \mathrm{C}$. The results of these numerical simulations are then compared with the corresponding previous ones, i.e. based on the calibrated parameters. The comparison has been done based on two quantities: (1) the total chloride concentration (weight percentage) $C$ at a clear cover of $15 \mathrm{~mm}$ (typical cover for stirrups in $\mathrm{RC}$ beams); (2) the depth of penetration $d_{p}$ measured from the exposure surface at which, the chloride concentration drops below a threshold of $0.01 \%$ (typical for fresh water). The numerical analysis results are shown in Table 4 together with their variations with respect to the results of the original simulations: at room temperature, $C=0.71 \%$ and $d_{p}=35.01 \mathrm{~mm}$, while at $38{ }^{\circ} \mathrm{C}, C=0.97 \%$ and $d_{p}=44.51 \mathrm{~mm}$. In general, all parameters show reasonable robustness, presenting mild changes as the parameters vary without any chaotic behavior. It is expected though that the response would be most sensitive to $B_{0}$ since it is the exponent of 10 in the Freundlich isotherm (Eq. 4). Nevertheless, the change in the concentration from the variation of $B_{0}$ is still within an acceptable range. This is a very important feature of this model making it very dependable despite the 
fact that it uses a limited number of free parameters.

\subsubsection{Representation of the discrete meso-scale features}

The importance of implementing this model in a discrete approach with a conduit transport network has its own clear merits. As it was shown in all simulation results, a small scatter around the average numerical simulation curve exists, which is due to the random geometry of the model representing the meso-scale heterogeneity of the material.

As shown in the slice in Fig. 8, chloride concentration is not radially varying in uniform contours yet it is very well representative of the experimental data and their scatter. To further explain this feature, Fig. 17 shows a vertical slice cut from the top of the modeled cylinder representing $\mathrm{C} 6$ at $50{ }^{\circ} \mathrm{C}$ after 48 days which corresponds to the chloride penetration profile shown in Fig. 10e. The location of the cut is schematically shown on the top right corner of the figure. The conduits (straight colored lines) are shown in Fig. 17a around the aggregate pieces (gray spheres) connecting the matrix space centers (colored small spheres). Colors show the $\mathrm{Cl} \%$ concentration along the conduits and inside the matrix spaces. Note that the color ranges are spaced on a log scale (see right color bar). By removing the aggregate (see Fig. 17b) concentration bands can be clearly seen and one can see the variations in colors along any horizontal line. To better see this variation, three zoomed locations are presented in Fig. 17c that clearly show the presence of two different chloride concentrations at the same depth (see orange and red in the middle picture) or even a lower concentration exiting above a higher concentration (yellow above orange in the left picture and dark blue above light blue in the right picture). Again, such local heterogeneity is an important feature of this model that can not be replicated with classical macroscopic continuum models which do not differentiate aggregate and matrix and treat concrete as a homogeneous material. Here the model is capable of representing the meso-scale tortuosity induced by relative aggregate sizes and spatial locations. It has to be noted here though that this only represents part of the heterogeneity since the conduit constitutive laws represent volume-averaged values of the matrix and aggregate properties, yet, once experimental data are made available in which chloride concentrations in both matrix and aggregate are differentiated, the model can be easily modified to account for this additional source of heterogeneity. 
a) Conduit transport network around the aggregate

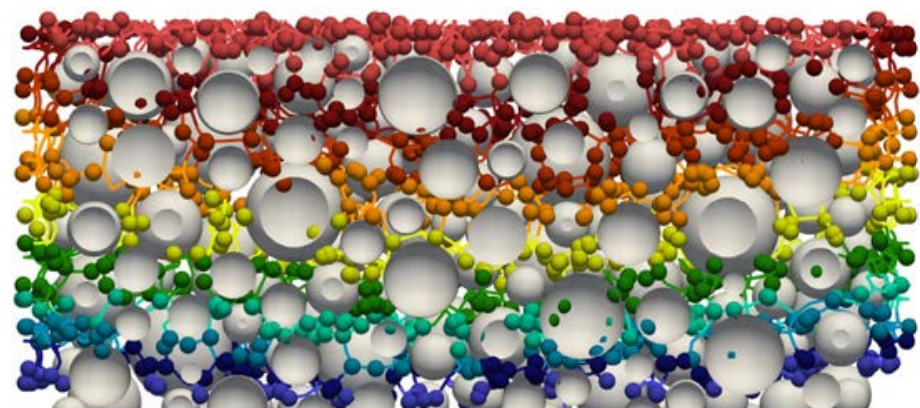

b) Conduit transport network with aggregate removed

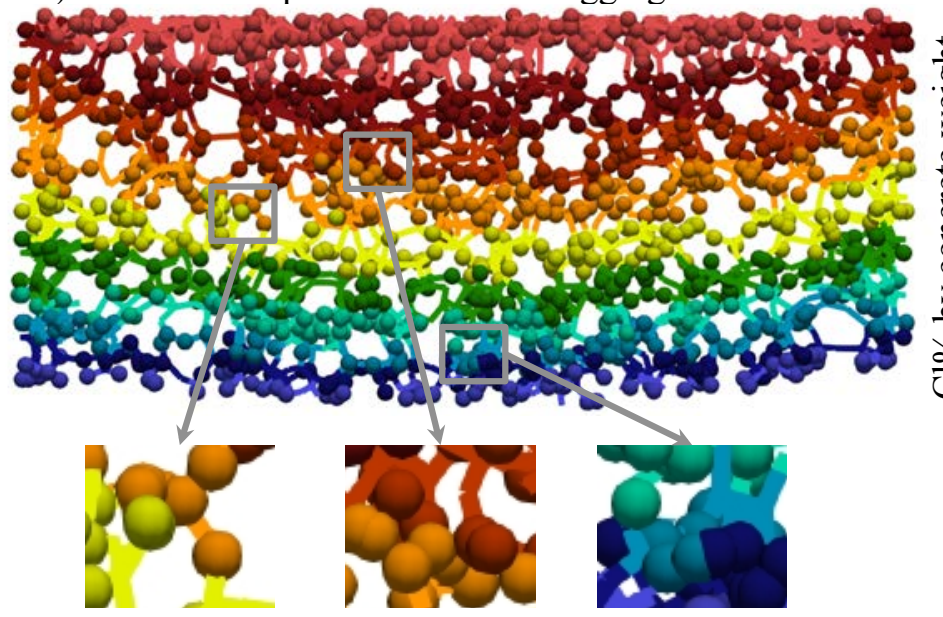

c) Matrix centers at same depth with different chloride concentrations
Slice

location
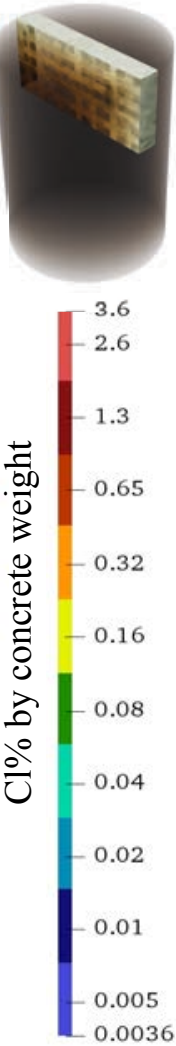

Figure 17: Conduit transport network ability to capture meso-scale heterogeneity in $\mathrm{Cl} \%$ concentration by concrete weight (a) Network between matrix space around aggregate (b) Aggregate removed, and (c) Zoomed locations within the network showing concentration differences

\section{Potential extension of the model}

Mimicking the internal meso-structure pathways makes it possible for the model to capture additional physics of the transport process if relevant comprehensive experimental data are available. First, the model can replicate the effects of meso-scale constrictivity if the volume assigned to each discrete conduit represents the matrix volume only (assuming impermeable aggregate), or if the different diffusivities are used for the aggregate and matrix. This will enable more accurate prediction of the local chloride concentration which is essential for explicit prediction of 
the location and size of anodic and cathodic areas on the rebar surface during early stages of chloride-induced corrosion.

A natural second extension of the model is to consider the effects of cracking on the diffusion process, and how that accelerates ingress of corrosive agents into the $\mathrm{RC}$ member which accelerates corrosion and then creates more cracking. In addition to corrosion, other sources of cracking found in the field include load application as well as drying and wetting or freeze/thaw cycles. Without accurate prediction of such processes, chloride penetration rates derived from controlled lab experiments would not predict accurately the actual field conditions. The proposed model has the potential to solve this problem through physical representation of cracking effects on diffusion rather than empirical representation and extrapolation. Note that the conduit network is located along the preferential locations for cracking and damage between aggregate pieces. This enables very realistic coupling between damage and diffusion where diffusivity can be updated based on the crack opening that is resolved explicitly down at the meso-scale, as done in modeling concrete thermal spalling during fire exposure [30] and moisture diffusion behavior of self-healing concrete [31].

\section{Conclusions}

This study presents a comprehensive multi-physics model calibrated and validated through experimental data to simulate the chloride transport in saturated and unsaturated cementitious materials. From the previous sections, the following main conclusions can be drawn:

(1) The proposed model can predict efficiently the chloride penetration in cementitious materials from cement paste to concrete under varying thermal, hygral and chloride concentration exposure conditions. Only 4 basic parameters are to be calibrated under isothermal conditions and only two additional parameters are needed to account for thermal effects. This is achieved by considering the main electro-chemical and physical processes in chloride transport as embedded mechanisms with predetermined parameters taken from previous experiments in the literature. 
(2) Coupling the chloride penetration with moisture diffusion, heat transfer and evolution of the cementitious material hydration reactions enables the framework to accurately represent effects of aging of the pore structure, effects of thermal gradients, and effects of hygral gradients (in unsaturated concrete).

(3) Ionic transport within the pore solution is represented by accounting for both pore solution chemistry (ionic activity and counter ion potentials) and pore solution concentration (ionic frictional/mechanical interactions).

(4) Effects of the pore structure on ionic transport are modeled by considering the effects of pore electric potential as well as the pore volume, tortuosity and constrictivity.

(5) Representing tortuosity and constrictivity as functions of the pore volume change is an effective simplification of model parameters that still enables the model to capture aging effects.

(6) Changes in pore structure due to aging are significant and important to consider when trying to correlate lab scale experiments done at young material ages to the behavior over prolonged time periods.

(7) By introducing thermal dependence into both chloride binding and diffusivity in pore solution, the model can capture the effect of temperature on chloride penetration in cementitous materials. The presented model is shown to be robust given its ability to represent a wide variety of conditions without significantly unexplained parameter variations, achieving a good balance between modeled physics and simplicity.

(8) Using a discrete conduit transport network enables capturing the meso-scale tortuosity which represents a part of the meso-scale heterogeneity effects on varying the chloride concentration. This is a very important feature for predicting the initiation and formation of micro-cell corrosion of reinforcement, which cannot be replicated using any classical macroscopic continuum model. 


\section{Acknowledgements}

The authors would like to acknowledge Prof. Tyler Ley at Oklahoma State University and Prof. Yunping Xi at University of Colorado at Boulder for providing helpful information regarding their experiments that we simulated in this work. Authors also would like to acknowledge the support from the Rensselaer Polytechnic Institute Center for Computational Innovations (CCI) to run the simulations in this paper using the High performance computing cluster. The second author gratefully acknowledges the support by the project Reshealience - Rethinking coastal defense and green-energy Service infrastructures through enHancEd-durAbiLity high-performance cement-based materials (EU Horizon H2020).

\section{References}

[1] G. Markeset, R. Myrdal, Modelling of reinforcement corrosion in concrete-state of the art, COIN Project report no7, SINTEF Building and Infrastructure (2008) 1891-1978.

[2] M. G. Barker, Life cycle costs of bridges: galvanized steel vs. concrete, Technical Report, US BRIDGE Cambridge, Ohio, 2018.

[3] L. Bertolini, B. Elsener, P. Pedeferri, E. Redaelli, R. B. Polder, Corrosion of Steel in Concrete, John Wiley \& Sons, Ltd, 2013.

[4] G. Di Luzio, L. Ferrara, M. C. A. y Alonso, P. Kuntz, V. Mechtcherine, C. Schroefl, Predicting the long-term performance of structures made with advanced cement based materials in extremely aggressive environments: current state of practice and research needs - the approach of h2020 project reshealience., in: E. Schlangen, G. de Schutter, B. S̈avija, H. Zhang, C. R. Rodriguez (Eds.), Proceedings of the international Symposium on Concrete Modelling (CONMOD2018), Delft, pp. 467-477.

[5] R. A. Patel, Q. T. Phung, S. C. Seetharam, J. Perko, D. Jacques, N. Maes, G. D. Schutter, G. Ye, K. V. Breugel, Diffusivity of saturated ordinary portland cement-based materials: A critical review of experimental and analytical modelling approaches, Cement and Concrete Research 90 (2016) 52-72.

[6] Y. Zhang, M. Zhang, Transport properties in unsaturated cement-based materials - a review, Construction and Building Materials 72 (2014) 367-379.

[7] S. Chatterji, Transportation of ions through cement based material. part 1: Fundamental equations and basic measurement techniques, Cement and Concrete Research 24 (1994) 907-912.

[8] S. Chatterji, Transportation of ions through cement based materials. part 2. adaptation of the fundamental equations and relevant comments, Cement and Concrete Research 24 (1994) 1010-1014. 
[9] S. Chatterji, Transportation of ions through cement based materials. part 3 experimental evidence for the basic equations and some important deductions, Cement and Concrete Research 24 (1994) 1229-1236.

[10] Z. P. Bažant, Physical model for steel corrosion in concrete sea structures theory, J. Struct. Div. ASCE 105 (ST6) (1979).

[11] T. Zhang, O. E. Gjørv, Diffusion behavior of chloride ions in concrete, Cement and Concrete Research 26 (1996) 907-917.

[12] E. Samson, J. Marchand, Numerical solution of the extended nernst-planck model, Journal of Colloid and Interface Science 215 (1999) 1-8.

[13] T. Luping, Concentration dependence of diffusion and migration of chloride ions part 1. theoretical considerations, Cement and Concrete Research 29 (1999) 1463-1468.

[14] T. Luping, Concentration dependence of diffusion and migration of chloride ions part 2. experimental evaluations, Cement and Concrete Research 29 (1999) 1469-1474.

[15] M. Masi, D. Colella, L. Bertolini, Simulation of chloride penetration in cement-based materials, Cement and concrete research 27 (1997) 1591-1601.

[16] Y. Xi, Z. P. Bažant, Modeling chloride penetration in saturated concrete, Cement and concrete research 11 (1999) $58-65$.

[17] B. Johannesson, K. Yamada, L.-O. Nilsson, Y. Hosokawa, Multi-species ionic diffusion in concrete with account to interaction between ions in the pore solution and the cement hydrates, Materials and Structures 40 (2007) 651.

[18] P. Nguyen, O.Amiri, Study of electrical double layer effect on chloride transport in unsaturated concrete, Construction and building materials 50 (2014) 492-498.

[19] P. Nguyen, O.Amiri, Study of the chloride transport in unsaturated concrete: Highlighting of the electrical double layer, temperature and hysteresis effects, Construction and building materials 122 (2016) 284-293.

[20] L.-Y. Li, J. Xia, S.-S. Lin, A multi-phase model for predicting the effective diffusion coefficient of chlorides in concrete, Construction and Building Materials 26 (2012) 295-301.

[21] J. Marchand, E. Samson, K. Snyder, J. Beaudoin, Modeling ion transport mechanisms in unsaturated porous media, Encyclopedia of Surface and Colloid Science 4 (2006) 3065-3074.

[22] E. Samson, J. Marchand, Modeling the effect of temperature on ionic transport in cementitious materials, Construction and building materials 37 (2007) 455-468.

[23] M. Isteita, Y. Xi, The effect of temperature variation on chloride penetration in concrete, Construction and Building Materials 156 (2017) 73-82.

[24] L. Homan, A. N. Ababneh, Y. Xi, The effect of moisture transport on chloride penetration in concrete, Construction and Building Materials 125 (2016) 1189 - 1195.

[25] J. Ožbolt, G. Balabanić, G. Periškic, M. Kušter, Modelling the effect of damage on transport processes in concrete, Construction and Building Materials 24 (2010) 1638-1648.

[26] J. Ožbolt, F. Oršanić, G. Balabanić, Modelling processes related to corrosion of reinforcement in concrete: coupled 
3d finite element model, Structure and Infrastructure Engineering 13 (2017) 135-146.

[27] J. Ožbolt, G. Balabanić, M. Kušter, 3d numerical modelling of steel corrosion in concrete structures, Corrosion Science 53 (2011) 4166-4177.

[28] L. Tang, L. O. Nilsson, Influence of cation type on diffusion behavior of chloride ions in concrete, Construction and Building Materials 99 (2015) 150-158.

[29] D. Conciatori, Étienne Grégoire, Éric Samson, J. Marchand, L. Chouinard, Sensitivity of chloride ingress modelling in concrete to input parameter variability, Materials and structures 48 (2014) 3023-3036.

[30] L. Shen, W. Li, X. Zhou, J. Feng, G. Di Luzio, Q. Ren, G. Cusatis, Multiphysics Lattice Discrete Particle Model for the simulation of concrete thermal spalling, Cement and Concrete Composites 106 (2020) 103457.

[31] F. Bousikhane, Experimental investigation and multi-physics computational modeling for assessment, mitigation and prevention of concrete deterioration, Ph.D. thesis, Northwestern University, 2018.

[32] M. Abdellatef, I. Boumakis, R. Wan-Wendner, M. Alnaggar, Lattice Discrete Particle Modeling of concrete coupled creep and shrinkage behavior: A comprehensive calibration and validation study, Construction and Building Materials 211 (2019) 629-645.

[33] M. Abdellatef, J. Vorel, R. Wan-Wendner, M. Alnaggar, Predicting Time Dependent Behavior of Post-Tensioned Concrete Beams: A Discrete Multiscale Multiphysics Formulation, Journal of Structural Engineering 145 (2019) 04019060 .

[34] M. Alnaggar, Multiscale Modeling of Aging and Deterioration of Reinforced Concrete Structures, Ph.D. thesis, Northwestern University, 2014.

[35] M. Alnaggar, M. Liu, J. Qu, G. Cusatis, Lattice Discrete Particle Modeling of acoustic nonlinearity change in accelerated alkali silica reaction (ASR) tests, Materials and Structures/Materiaux et Constructions 49 (2016).

[36] M. Alnaggar, G. Di Luzio, G. Cusatis, Modeling time-dependent behavior of concrete affected by Alkali Silica Reaction in variable environmental conditions, Materials 10 (2017).

[37] E. Masoero, G. Cusatis, G. Di Luzio, C-S-H gel densification: The impact of the nanoscale on self-desiccation and sorption isotherms, Cement and Concrete Research 109 (2018) 103 - 119.

[38] G. Di Luzio, G. Cusatis, Hygro-thermo-chemical modeling of high performance concrete. I: Theory, Cement and concrete composite 31 (2009) 301-308.

[39] G. Di Luzio, G. Cusatis, Hygro-thermo-chemical modeling of high-performance concrete. II: Numerical implementation, calibration, and validation, Cement and concrete composite 31 (2009) 309-324.

[40] J. Bear, Y. Bachmat, Introduction to modelling of transport phenomena in porous media., Dordrecth: Kluwer Academic Publishers, 1990.

[41] E. Samson, J. Marchand, K. Snyder, J. Beaudoin, Modeling ion and fluid transport in unsaturated cement systems in isothermal conditions, Cement and Concrete Research 35 (2005) 141 - 153.

[42] E. Samson, J. Marchand, Modeling the transport of ions in unsaturated cement-based materials, Computers \& Structures 85 (2007) $1740-1756$. 
[43] E. Samson, J. Marchand, J. Beaudoin, Describing ion diffusion mechanisms in cement-based materials using the homogenization technique, Cement and Concrete Research 29 (1999) 1341 - 1345.

[44] Q. Yuan, C. Shi, G. D. Schutter, K. Audenaert, D. Deng, Chloride binding of cement-based materials subjected to external chloride environment - a review, Construction and Building Materials 23 (2009) 1-13.

[45] T. Luping, L.-O. Nilsson, Chloride binding capacity and binding isotherms of opc pastes and mortars, Cement and concrete research 23 (1993) 247-253.

[46] P. Spiesz, M. Ballari, H. Brouwers, Rcm: A new model accounting for the non-linear chloride binding isotherm and the non-equilibrium conditions between the free- and bound-chloride concentrations, Construction and Building Materials 27 (2012) $293-304$.

[47] A. Dousti, R. Rashetnia, B. Ahmadi, M. Shekarchi, Influence of exposure temperature on chloride diffusion in concretes incorporating silica fume or natural zeolite, Construction and Building Materials 49 (2013) 393 - 399.

[48] A. Dousti, M. Shekarchi, Effect of exposure temperature on chloride-binding capacity of cementing materials, Magazine of Concrete Research 67 (2015) 821-832.

[49] D. Panesar, S. Chidiac, Effect of cold temperature on the chloride-binding capacity of cement, Journal of Cold Regions Engineering 25 (2011) 133-144.

[50] O. Wowra, M. Setzer, M. Setzer, R. Auberg, Sorption of chlorides on hydrated cements and c3s pastes, Frost resistance of concrete (1997) 147-153.

[51] H. Zibara, Binding of external chlorides by cement pastes, Ph.D. thesis, National Library of Canada= Bibliothèque nationale du Canada, 2001.

[52] M. Roberts, Effect of calcium chloride on the durability of pre-tensioned wire in prestressed concrete, Magazine of Concrete Research 14 (1962) 143-154.

[53] M. Maslehuddin, C. Page, C. Rasheeduzzafar, A. Al-Mana, Effect of temperature on pore solution chemistry and reinforcement corrosion in contaminated concrete, SPECIAL PUBLICATION-ROYAL SOCIETY OF CHEMISTRY 183 (1996) 67-75.

[54] K. Norling-Mjörnell, A model on self-desiccation in high-performance concrete., in: self-desiccation and its importance in concrete technology, Proceedings of the international research seminar, Lund, Sweden, pp. 141157.

[55] M. Rangelov, S. Nassiri, Empirical time-dependent tortuosity relations for hydrating mortar mixtures based on modified archie's law, Construction and Building Materials 171 (2018) 825-838.

[56] H. F. W. Taylor, Cement Chemistry, Thomas Telford, London, 1997.

[57] V. S. Bagotsky, Fundamentals of electrochemistry, Wiley, 2005.

[58] J.-Z. Zhang, N. Buenfeld, Presence and possible implications of a membrane potential in concrete exposed to chloride solution, Cement and Concrete Research 27 (1997) 853-859.

[59] E. Samson, G. Lemaire, J. Marchand, J. Beaudoin, Modeling chemical activity effects in strong ionic solutions, Computational Materials Science 15 (1999) 285-294. 
[60] J. F. Pankow, Aquatic chemistry concepts, CRC Press, 2018

[61] H. Justnes, E. Rodum, Chloride ion diffusion coefficients for concrete - a review of experimental methods, 10th International Congress on the Chemistry of Cement, Gothenburg, IV (1997) 8.

[62] G. Akerlof, G. Kegeles, Thermodynamics of sodium hydroxide solutions, Journal of the American Chemical Society 62 (1940) $620-640$.

[63] A. Neville, Electrolyte Solutions, Butterworths, London, 1959.

[64] K. A. MacDonald, D. O. Northwood, Experimental measurements of chloride ion diffusion rates using a twocompartment diffusion cell: Effects of material and test variables, Cement and Concrete Research 25 (1995) 1407-1416.

[65] A. Asbridge, G. Chadbourn, C. Page, Effects of metakaolin and the interfacial transition zone on the diffusion of chloride ions through cement mortars, Cement and Concrete Research 31 (2001) 1567-1572.

[66] J. Arsenault, J. Bigas, J. Ollivier, Determination of chloride diffusion coefficient using two different steady-state methods: Influence of concentration gradient, in: Proc. of the Int. RILEM Workshop, St. Remy les Chevreuse, France, pp. 150-160.

[67] T. Zhang, Chloride diffusivity in concrete and its measurement from steady state migration testing, Norwegian University of Science and Technology, 1997.

[68] S. Chatterji, Transportation of ions through cement based materials. part 1 fundamental equations and basic measurement techniques, Cement and Concrete Research 24 (1994) 907-912.

[69] J. Newman, K. E. Thomas-Alyea, Electrochemical system, Wiley, 1991.

[70] Z. Liu, Y. Zhang, Q. Jiang, Continuous tracking of the relationship between resistivity and pore structure of cement pastes, Construction and Building Materials 53 (2014) 26-31.

[71] P. Carman, Fluid flow through granular beds, Chemical engineering research and design 75 (1997) S32-S48.

[72] M. Pathirage, D. Bentz, G. Di Luzio, E. Masoero, G. Cusatis, The ONIX model: a parameter-free multi-scale framework for the prediction of self-desiccation in concrete, Cement and Concrete Composites 103 (2019) 36-48.

[73] F. J. Ulm, O. Coussy, Modeling of thermo-chemical-mechanical couplings of concrete at early age, Journal of Engineering Mechanics 121 (1995) 785-794.

[74] M. Cervera, J. Oliver, T. Prato, Thermo-chemical-mechanical model for concrete. i: Hydration and aging, Journal of Engineering Mechanics 125 (1999) 1018-1027.

[75] D. Gawin, F. Pesavento, B. A. Schrefler, Hygro-thermo-chemo-mechanical modelling of concrete at early ages and beyond, part i: hydration and hygro-thermal phenomena, International Journal for Numerical Methods in Engineering 67 (2006) 299-331.

[76] G. Di Luzio, G. Cusatis, Solidification-Microprestress-Microplane (SMM) theory for concrete at early age: Theory, validation and application, International Journal of Solids and Structures 50 (2013) 957 - 975.

[77] M. Alnaggar, G. Cusatis, G. Di Luzio, Lattice Discrete Particle Modeling (LDPM) of Alkali Silica Reaction (ASR) deterioration of concrete structures, Cement and Concrete Composites 41 (2013). 
[78] G. Di Luzio, L. Ferrara, V. Krelani, Numerical modeling of mechanical regain due to self-healing in cement based composites, Cement and Concrete Composites 86 (2018) $190-205$.

[79] M. Pathirage, F. Bousikhane, M. D’Ambrosia, M. Alnaggar, G. Cusatis, Effect of alkali silica reaction on the mechanical properties of aging mortar bars: Experiments and numerical modeling, International Journal of Damage Mechanics (2018).

[80] G. Cusatis, D. Pelessone, A. Mencarelli, Lattice discrete particle model (LDPM) for failure behavior of concrete. i: Theory, Cement and Concrete Composites 33 (2011) 881-890.

[81] G. Cusatis, A. Mencarelli, D. Pelessone, J. Baylot, Lattice discrete particle model (LDPM) for failure behavior of concrete. ii: Calibration and validation, Cement and Concrete Composites 33 (2011) 891-905.

[82] F. Bousikhane, W. Li, G. Di Luzio, G. Cusatis, Full coupling between diffusion and mechanical analysis in a discrete computational framework, in: G. Meschke, B. Pichler, J. G. Rots (Eds.), Proceedings of the Conference on Computational Modelling of Concrete and Concrete Structures (EURO-C 2018), CRC Press Book, Bad Hofgastein, Austria, 2018, pp. 305-310.

[83] P. D., MARS: modeling and analysis of the response of structures-user's manual, ES3,Beach(CA), 2009.

[84] Z. Bažant, L. Najjar, Nonlinear water diffusion in nonsaturated concrete, Matériaux et Construction 5 (1972) 3-20.

[85] Z. P. Bažant, M. F. Kaplan, Concrete at high temperatures: material properties and mathematical models, Longman, 1996.

[86] A. Neville, Properties of concrete, John Wiley and Sons, New York, 1997.

[87] M.Relis, I.Soroka, Variation in density of portland cement hydration products, Cement and Concrete Research 7 (1977) 1092-1097.

[88] M. K. Moradllo, M. T. Ley, Quantitative measurement of the influence of degree of saturation on ion penetration in cement paste by using X-ray imaging, Construction and Building Materials 141 (2017) 113-129.

[89] M. K. Moradllo, Q. Hu, M. T. Ley, Using X-ray imaging to investigate in-situ ion diffusion in cementitious materials, Construction and Building Materials 136 (2017) 88-98.

[90] M. Alnaggar, G. Cusatis, Automatic parameter identification of discrete mesoscale models with application to the coarse-grained simulation of reinforced concrete structures, in: 20th Analysis and computation specialty conference, pp. 406-417.

[91] M. Alnaggar, N. Bhanot, A machine learning approach for the identification of the lattice discrete particle model parameters, Engineering Fracture Mechanics 197 (2018) 160-175.

[92] ASTM C1152 / C1152M-04(2012)e1, Standard Test Method for Acid-Soluble Chloride in Mortar and Concrete, 2012.

[93] H. Y. Moona, H. S. Kim, Relationship between average pore diameter and chloride diffusivity in various concretes, Construction and building materials 20 (2006) 725-732. 